\title{
DEL PASSAGe A LAS GaLERÍAS POPULARES
}

WILEY LUDEÑA DIANA TORRES
PROFESOR, UNIVERSIDAD NACIONAL DE INGENIERÍA, LIMA, PERÚ. PROFESORA, PONTIFICIA UNIVERSIDAD CATÓLICA DEL PERÚ, LIMA, PERÚ.
Aunque resulte descriptiva y elemental en $\mathbf{1}$ su formulación, la definición del passage efectuada por la Guía Ilustrada de París (1852) resulta precisa: "son galerías cubiertas de cristal y revestidas de mármol que atraviesan edificios enteros, cuyos propietarios se han unido para tales especulaciones. A ambos lados de estas galerías, que reciben la luz desde arriba, se alinean las tiendas más elegantes, de modo que un pasaje semejante es una ciudad, e incluso un mundo en pequeño, en el que el comprador ávido encontrará todo lo que necesita" (en Benjamin, 2004, p. 69). Johann Friedrich Geist ha logrado sistematizar la gran diversidad de tipos de pasajes. Desde aquellos de un solo corredor recto perpendicular, hasta aquellos organizados en cruz en relación al número de corredores. O desde los pasajes-corredor hasta los pasajes-corredor/ plaza o pasajes/plaza, pasando por edificaciones de cuerpo en ángulo, dos ángulos o más, así como pasajes de uno, dos y cuatro ingresos/fachadas, entre otros tipos (Geist, 1989). Dependiendo del país, la lengua y ciertas tradiciones, el término francés passage ha sido traducido como pasaje, arcade,

galerie, galería, passaz y galleria, entre otros. En el caso peruano, sin embargo, la débil institucionalización del passage ha producido un significado ambiguo para el término, el cual se identifica más que con una edificación de uso comercial, con un tipo de calle angosta de carácter peatonal y uso casi exclusivamente residencial. En el presente texto se empleará la denominación de pasaje en el sentido de su acepción comercial clásica.

Refiriéndonos a una mezcla andino-urbana 2 de cualquier estilo, función o destino.

Resultan acertadas las diferencias que 3 establece Kurt Weyland (1997) entre las nociones clásicas de liberalismo y populismo, y aquellas definidas como neoliberalismo y neopopulismo. A diferencia del liberalismo clásico que se aplicó creando al mismo tiempo un Estado a la medida, el neoliberalismo apuesta por el desmontaje del aparato estatal por medio de la absolutización de las leyes del libre mercado y la privatización de todo el aparato productivo. A diferencia del populismo clásico de mediados del siglo Xx que promovió la modernización industrial y tomó como su base social al emergente proletariado industrial latinoamericano, el neopopulismo de la década de 1990 detenta un programa y referente social distintos: apuesta por políticas de libre mercado y por la transnacionalización económica. Se opone a formas organizadas de la sociedad civil y convoca con retórica populista al sector informal y la masa de pobres en situación extrema como su principal base social. En términos de Weyland, Fujimori es un típico neopopulista neoliberal (Weyland, 1997).
O DEL LIBERALISMO CRIOLLO A LA CIUDAD NEOLIBERAL

$\mathbf{R}$ efiriéndose a Charles Fourier en París y a la transfiguración de los passages en un auténtico falansterio idílico, Walter Benjamin evoca al país de Jauja, aquella leyenda medieval de un país mitológico feliz, autosuficiente, saludable y en éxtasis permanente (1982). Pero la Jauja peruana no es completamente la Jauja mítica, ni los pasajes de Lima son esa colosal maquinaria humana que supone el sueño de Fourier ${ }^{1}$.

En 1924 cuando se inaugura el pasaje Carmen -el primer passage en Lima- el célebre Passage des Panoramas, uno de los primeros de París, cumplía 125 años de construido mientras que el Passage de L'Opera, el epicentro emblemático del París de los surrealistas y Louis Aragon, era demolido luego de más de un siglo de existencia. Diez años antes de esta fecha, en 1914, Buenos Aires ya contaba con pasajes de una envergadura y esplendor en algunos casos más ostentosos que sus similares europeos, como la lujosa galería Bom Marché (1890) o la no menos imponente galería Güemes (1913-1915).

De este periodo fundacional quedan numerosos pasajes en algunas de las grandes capitales de Latinoamérica. La mayoría de estos han desaparecido, están en trance de demolición, han sido reconvertidos o aún se encuentran vigentes. En contraste, en algunas metrópolis como Lima se ha producido en las últimas dos décadas un fenómeno tanto explosivo como vital y caótico: la expansión de una versión $c h i$ $c h a^{2}$ del pasaje decimonónico bajo el formato de galería comercial popular. Dos momentos y dos encuadres contrapuestos de arquitecturas de consumo que se desprenden de los ciclos de una misma historia y economía política: la del liberalismo del siglo xIX y el neoliberalismo populista del siglo XXI, con sus respectivas expresiones en términos de ciudad y arquitectura ${ }^{3}$.

Los pasajes no son un invento parisino ya que sus antecedentes, como una modalidad de comercio y tipo edilicio, se encuentran en el bazar oriental. A decir de Walter Benjamin, el pasaje, concebido como el templo del capital mercantil, es producto de las entrañas de la ciudad del capitalismo liberal del siglo $\mathrm{XIX}^{4}$. Del mismo modo que en la actualidad, en el hormigueo irredento de la ciudad neoliberal, las galerías comerciales representan en muchos sentidos a ese casino comercial sin templo ni ciudad que reproduce sin pausa la voracidad de esa vuelta al capitalismo salvaje traducido en neoliberalismo neo populista agresivo y descreído de la ciudad y en cualquier forma de ciudadanía.
Si para el liberalismo decimonónico los passages recreaban un mundo como complemento onírico de la ciudad con pasantes embrujados y ciudadanos en cierne, para el neoliberalismo neo populista las galerías agotan este mundo en sí, convirtiendo a la ciudad en un subproducto deleznable del imperativo consumista. Si la ciudad liberal del siglo xIx inventó el passage y la ciudad posliberal del siglo xx propuso el civic center, la ciudad del reajuste neoliberal y neo populista en países periféricos de los centros de comando global masificó las galerías comerciales como una expresión visceral de las dimensiones más ominosas y/o positivas de aquello que Hernando de Soto denomina como capitalismo popular.

A diferencia de otras grandes capitales del continente, Lima no es una ciudad de passages surgidos como un eco omnipresente del siglo xix y la Belle Époque europea. Pero en sentido inverso y en contraste a estas ciudades, la capital peruana es probablemente el ejemplo más extremo en Latinoamérica de cómo el reajuste neoliberal de inicios de la década de 1990 ha generado una explosión sin límites de galerías comerciales y otras derivaciones populares del antiguo pasaje decimonónico. Paradoja que se encuentra en medio de una sola historia en términos de arquitectura y economía política: a menos pasajes de ayer, más galerías populares de hoy. O expresado de otra manera: a menos liberalismo económico de ayer, menos pasajes y a más neoliberalismo populista, más galerías populares.

El pasaje, concebido como ese artefacto icónico del capitalismo del siglo XIX, arribó al Perú recién en la segunda década del siglo xx como símbolo de una modernidad aún esquiva en medio de una ciudad estructuralmente anti-moderna. Por ello, Lima ha sido para esta máquina de sueños como ese campesino de Louis Aragon en Le paysan de Paris (1926), una ciudad provinciana e ingenua que se deslumbra ante lo nuevo que no produce; una ciudad que no inventa por sí misma los pasajes, sino que los acoge para maravillarse y escenificar la experiencia de una vida ajena.

Es esta distancia y desarraigo esencial la que envuelve el origen y la historia del pasaje limeño. Marca una historia de fijación banal por lo nuevo, pero al mismo tiempo produce una indiferencia social, también de modernidad súbita y obsolescencia prematura. Un ejemplo de esto es el pasaje Carmen, el primer pasaje edificado en Lima y que hoy sobrevive con tiendas y quioscos precarios para la venta de postales y baratijas. Del mismo modo, el pasaje Ronald (1929), construido en el puerto del Callao, un auténtico 
Traducción capitalista del bazaar, el passage se instala en el siglo xIx como expresión imprescindible de la ciudad moderna, hoy superado por el mall. Sin embargo, las políticas neoliberales latinoamericanas han reinstalado sus versiones recientes como símbolo del capitalismo popular.

PALABRAS CLAVE: ARQUITECTURA - PERÚ, TEJIDO URBANO, ARQUITECTURA COMERCIAL, PASAJES, CIUDAD NEOLIBERAL.

homenaje a la mejor tradición arquitectónica de los pasajes europeos, nunca funcionó plenamente como tal y hoy es casi un extraño cuerpo fantasma que pretende resucitar de vez en cuando. Acontece lo mismo con los dos pasajes modernos de Lima: la galería Boza (1956) y la galería Gallos-Mogollón (1959).

Tal vez en esta contradicción y compleja notación arquitectónica, urbana y cultural resida la magia de esta serie de primeros y únicos pasajes construidos en la Lima oligárquica y conservadora del siglo xx. Cada uno con una historia que no deja de ser igualmente surrealista, tal como el intensivo y colorido uso cotidiano que hoy acogen en medio de un paisaje degradado y fantasmagórico. Pero esta es una historia que empezó a cambiar dramáticamente desde hace dos décadas al producirse el drástico reajuste neoliberal y neo populista promovido por el gobierno de Alberto Fujimori (1990-2000). Si bien antes de este hecho se produciría una especie de invasión de ambulantes y campos feriales en el centro de Lima en su expresión más miserable y precaria, es a partir de fines de mediados de la década de 1990 que se experimentaría una auténtica explosión y refinamiento cutre de lo que en el Perú se denominan las galerías comerciales de corte popular.

Este fenómeno es la expresión del nuevo ciclo de expansión económica que experimenta el Perú tras el reajuste estructural y la consiguiente vuelta a formas primarias de capitalismo salvaje. Ahí están las decenas de nuevas galerías comerciales erigidas los últimos años en los barrios de comercio más conocidos de Lima: la zona del Mercado central y los barrios comerciales Gamarra, Las Malvinas y avenida Abancay, entre otros. Las galerías comerciales de hoy son los pasajes de ayer. Recrean los fundamentos tipológicos del bazar y del pasaje decimonónico, pero no son más que un templo sacralizado del capitalismo mercantil. Son el nuevo casino comercial estridente y soporífero diseñado al mismo tiempo por el capitalismo tardío global y el consenso de Washington para los países de la periferia y los millares de consumidores pobres.

\section{LOS PASSAGES DEL LIBERALISMO CRIOLLO}

El pasaje Carmen, primer pasaje limeño, abrió sus puertas en 1924 en medio de las celebraciones del primer centenario de la Independencia y de la batalla de Ayacucho. Es un acontecimiento que forma parte de la fiesta nacional. El pasaje Ronald, el segundo pasaje de la década, se inauguró en 1929 casi en las postrimerías del gobierno de Augusto B. Leguía (1919-1930), autoproclamado como el gobierno de la Patria nueva.
A kind of capitalist bazaar, the passage is installed in the $19^{\text {th }}$ century as an essential expression of the modern city, now superseded by the mall. However, Latin American neoliberal policies have reinstated its newer versions as a symbol of popular capitalism

KEYWORDS: ARCHITECTURE - PERU, URBAN FABRIC, COMMERCIAL ARCHITECTURE, PASSAGES, NEOLIBERAL CITY. English text in p. 48
¿Cómo es posible que una construcción de consumada ortodoxia Beaux Arts como el pasaje Carmen surja en una Lima que aspiraba a dejar atrás cualquier señal de arquitectura decimonónica y eclecticismo historicista conservador en ese momento? Y segundo, ¿cómo se entiende que una edificación como el pasaje Ronald se construya como un enclave futurista de gran despliegue cuando la prosperidad del oncenio leguiísta ya era cosa del pasado y nada justificaba tal desmesura?.

En realidad, los pasajes estuvieron en el imaginario urbano de algunos limeños ilustrados con experiencia de vida europea desde el último cuarto del siglo XIX. Que el primero se haya construido casi medio siglo después se debe fundamentalmente a dos factores: el periodo de depresión económica que produjo la Guerra del Pacífico (1879-1883), y la ausencia de transformaciones estructurales en la ciudad. Hecho que recién empezaría a producirse a inicios del siglo $\mathrm{xx}$ al retomarse el proyecto modernizador de reestructuración haussmanniana de Lima formulado por José Balta, Nicolás de Piérola y Luis Sada entre 1868 y 1872.

A diferencia de la experiencia europea en la que los pasajes se adelantaron a los grandes almacenes, en Lima sucedió lo contrario. Este hecho influiría de manera decisiva en el destino de los pasajes y su débil institucionalización, así como en su eventual fracaso económico comercial. En 1917, cuando el inmigrante alemán Augusto Fernando Oechsle inaugura al borde de la Plaza Mayor la primera gran tienda moderna por departamentos de Lima, los pasajes del futuro se hicieron de un porvenir incierto. La primera víctima: el pasaje Carmen ubicado a menos de $200 \mathrm{~m}$ de la Casa Oechsle.

En una sociedad empobrecida sin clase media y con una oligarquía conservadora, cerrada y frontalmente distante de esa clase media emergente llena de huachafos y huachafitas, como señala David S. Parker, los espacios públicos o semipúblicos como los cafés, restaurantes y pasajes jamás lograron un grado de empatía y legitimidad social con la alta sociedad limeña. Los espacios privilegiados por la élite constituían un mundo autorreferencial como los clubes o negocios cerrados similares a la Casa Oechsle, protegidos de los infiltrados sociales (Parker, 1998).

\section{EL PASAJE CARMEN Y LA EVOCACIÓN TARDÍA DE LA REPÚBLICA ARISTOCRÁTICA}

El pasaje Carmen se constituye como uno de los componentes singulares de un conjunto de tres edificaciones estructuradas a partir de la Casa de Correos y 
FIG 1 A FIG 6

Pasaje El Carmen, Centro Histórico de Lima, Perú. 1924

El Carmen Gallery, Historical center of Lima, Perú, 1924.

FIG 1

Planta de emplazamiento. E. 1: 7.500 Levantamiento: Diana Torres Obregón. Site plan. Scale 1: 7.500

Survey: Diana Torres Obregón.

\section{FIG 2 A FIG 4}

Fotografías: Wiley Ludeña Urquizo. Photography: Wiley Ludeña Urquizo.

FIG 5

Planta general. E. 1: 1.000. Levantamiento: Diana Torres Obregón. General plan. Scale 1: 1.000. Survey: Diana Torres Obregón.

FIG 6

Corte longitudinal. E. 1: 1.000

Levantamiento: Diana Torres Obregón.

Long section. Scale 1: 1.000

Survey: Diana Torres Obregón.

FIG 7 A FIG 10

Pasaje Ronald, Centro Histórico de Lima, Perú, 1929

Ronald Gallery, Historical center of Lima, Perú, 1929.

FIG 7 Y FIG 8

Fotografías: Wiley Ludeña Urquizo. Photography: Wiley Ludeña Urquizo.

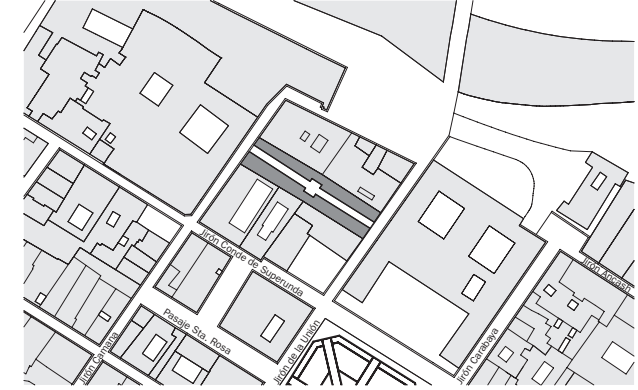

FIG 1

Telégrafos (1897). Se encuentra a mitad de una manzana ubicada en una zona estratégica y privilegiada del centro de Lima: junto al Palacio de Gobierno y cerca de una esquina de la Plaza Mayor. El pasaje, con una longitud de $115 \mathrm{~m}$ y $6 \mathrm{~m}$ de ancho, une los jirones Camaná y de La Unión (fig. 1). Fue diseñado por el arquitecto Raúl María Pereira y construido en 1924.

El pasaje, que en orden de aparición es la tercera edificación del conjunto, fue concebido como un componente integrado con la arquitectura académica de Emilio Parzo y Máximo Doig, responsables del diseño de la Casa de Correos y Telégrafos, considerado como uno de los ejemplos más acabados de arquitectura Beaux Arts de la Lima finisecular. Con la voluntad de irradiar una manifiesta monumentalidad ${ }^{5}$ a partir de las portadas de ingreso, la arquitectura del pasaje Carmen, en sus dos plantas, recoge con coherencia compositiva los fundamentos tipológicos de una tradición edilicia perfectamente decantada para ese entonces (figs. 2 y 4 ). comercial o cultural en la historia de la Lima del siglo xx. Funcionó en el tiempo como un espacio básicamente tributario de las actividades propias y conexas al mundo postal y telegráfico. Sin embargo, también se hizo de comercios, restaurantes o bodegas que en su mejor época -la del oncenio de Leguía y años posteriores- acogieron a parte de la élite limeña y mucho más a la burocracia gubernamental.

\section{EL PASAJE RONALD 0 EL SUEÑO PERDIDO DE LA PATRIA NUEVA}

El pasaje Ronald empezó a ser construido en 1923 y fue inaugurado recién en 1929, el mismo año del crack de Nueva York y pocos meses antes de la debacle del oncenio leguiísta. Tal vez esto explique su opaco destino: nunca alcanzó el esplendor soñado por su propietario. El concepto y diseño corresponden decididamente a la voluntad personal de Guillermo Ronald, quien deseaba reproducir en el Callao el formato y estética de las arcades más refinadas y monumentales que había frecuentado en Europa. En el diseño y construcción participaron Nicolaus Babinski, arquitecto austrohúngaro radicado en Lima, y el ingeniero inglés Bunting (Coello Pohl, 2009; Dávila, 2013)

El edificio atraviesa una manzana irregular, uno de cuyos lados hace frente a la plaza de la iglesia matriz. Las dos puertas de acceso del pasaje, de $6 \mathrm{~m}$ de ancho y $55 \mathrm{~m}$ de largo, vinculan los jirones Constitución e Independencia. El lote y edificio que lo ocupa tiene

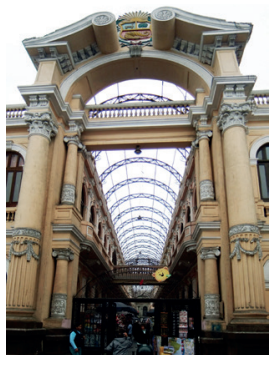

FIG 2

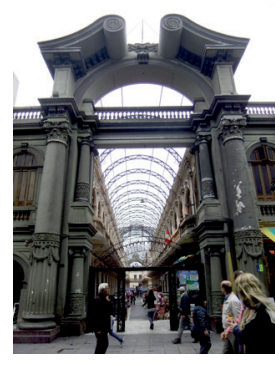

FIG 3

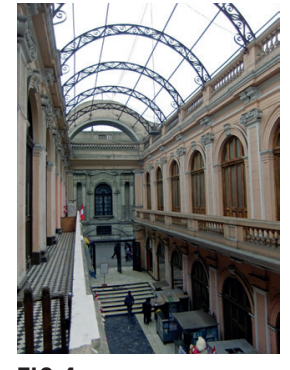

FIG 4

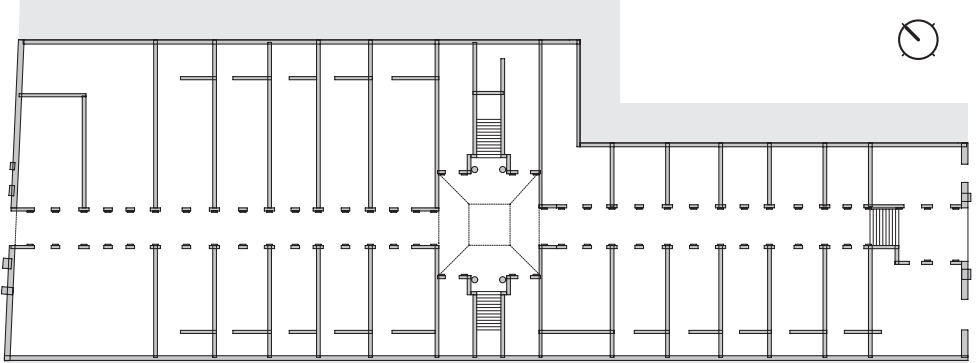

FIG 5
Después del terremoto de 1970 la cubierta 5 de vidrio fue retirada por razones de seguridad lo que ha otorgado a la arquitectura del pasaje de una singular pátina de máquina abandonada sin serlo. El techo desvestido se ha hecho un símbolo de las incongruencias de una arquitectura concebida para el frío y la lluvia en un lugar donde nunca llueve ni hace frío.
A lo largo, el corredor central está cubierto por una bóveda de cañón con techo de vidrio. Dicha bóveda se convierte en una estructura de aristas y desarrollo estructural particular para cubrir el patio central, adjudicándose así una visible monumentalidad. La estructura de hierro de la cubierta marca el ritmo de una secuencia estructural que se compone desde los sótanos del pasaje (figs. 3, 5 y 6). Como ha caracterizado José García Bryce, "la arquitectura del pasaje se estructura a partir del repertorio neorrenacentista de las fachadas interiores del pasaje y la notación barroca de los modillones, balaustradas, las órdenes de las columnas y las pilastras" (García Bryce, 1967, p. 180). Las portadas de ingreso de ambos lados son un indiscutible factor de representación del pasaje. Se componen a partir de un elaborado despliegue de componentes historicistas neoclásicos dotados de una monumentalidad con cierto dramatismo barroco. Portadas que sacralizan un espacio profano por naturaleza.

El pasaje Carmen no se convirtió en el lugar mítico de encuentro ni en el símbolo de una épica un ancho de $30 \mathrm{~m}$ (fig. 7). El volumen resultante es un enorme artefacto de seis plantas cuyas proporciones debieron parecer superlativas en el contexto urbano contiguo, configurado hasta la actualidad por edificaciones de dos pisos como promedio. Junto a la Casa Wiese (1922) y el edificio Gildemeister (1928), ambos de seis pisos y ubicados en el centro de Lima, el pasaje Ronald es otro de los primeros rascacielos de Lima. Contaba con más de noventa locales para oficinas y negocios en sus seis plantas, así como doce departamentos que desde el inicio albergaron a prominentes familias de la colonia inglesa del puerto. En el quinto piso se ubicaba un célebre bar inglés conocido como Twin Deck. Sus lujosos ascensores fueron los primeros en instalarse en el Callao.

Junto a ello, el pasaje del primer piso resulta de manifiesta solemnidad y gran espacialidad. Esta se ve reforzada tanto por el mármol de los pisos y escaleras, los enchapes de madera y los frisos, como por los capiteles jónicos, medallones y otros detalles diseñados bajo los principios de un cierto neoclasicismo evocado 


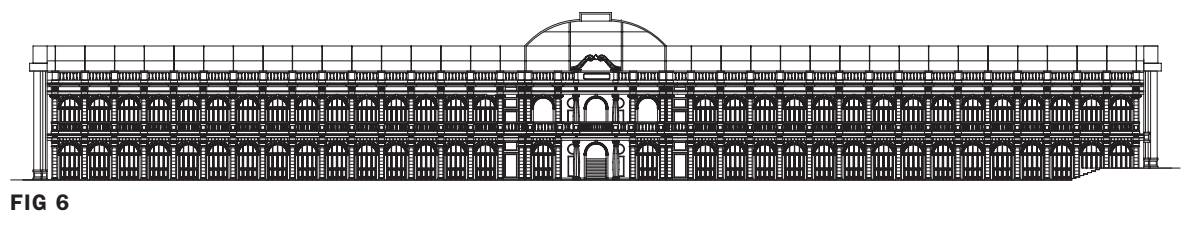

FIG 9 Levantamiento: Diana Torres Obregón. Site plan. Scale 1: 7.500. Survey: Diana Torres Obregón.

\section{FIG 10}

Elevación principal. E. 1: 500. Levantamiento: Diana Torres Obregón. Main elevation. Scale 1: 500 Survey: Diana Torres Obregón.

\section{FIG 11 A FIG 13}

Galería Boza, Centro Histórico de Lima, Perú, 1956

Boza Gallery, Historical center of Lima, Perú, 1956

\section{FIG 11}

Planta primer nivel. E. 1: 1.000.

Levantamiento: Diana Torres Obregón.

First floor plan. Scale 1: 1.000.

Survey: Diana Torres Obregón.

en clave moderna. El corredor comercial de doble su configuración morfológica (figs. 9 y 10).
Planta de emplazamiento. E. 1: 7.500. altura se encuentra cubierto en la parte central por un techo de vidrio de dos aguas. Aquí, la luminosidad cenital adquiere un particular efecto debido a la cobertura hecha por vitrales artísticamente elaborados y dispuestos a modo de nave (fig. 8). En el pasaje Ronald la idea del edificio pasaje se hace evidente desde el exterior. El edificio es el pasaje y este en su estructura interior expresa la voluntad del edificio como arquitectura y como mundo abigarrado de múltiples actividades. Es decir, un edificio ciudad. Al erigirse de cara al puerto del Callao, el edificio se transformó no solo en un hito incontrovertible, sino en una especie de faro mercantil que domina el perfil del puerto y marca

En su mejor y corta época el pasaje Ronald fue el epicentro de lo más moderno y europeo del puerto del Callao. Era el punto de encuentro de empresarios, oficiales de mar y numerosas familias de ascendencia europea que residían en el barrio La Punta o en los departamentos de los pisos superiores del mismo pasaje. Con el tiempo sus oficinas empezaron a ser ocupadas por diversas instituciones administrativas, deportivas o algunas relacionadas con las empresas navieras. Se instalaron oficinas de abogados, ingenie-

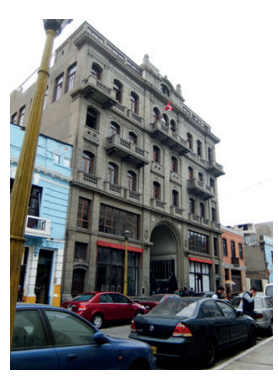

FIG 7

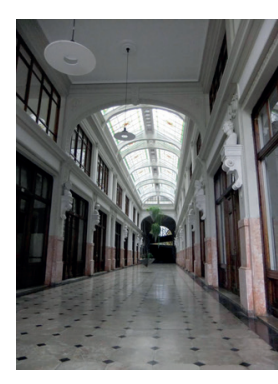

FIG 8

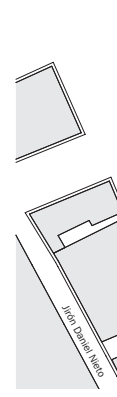

FIG 9

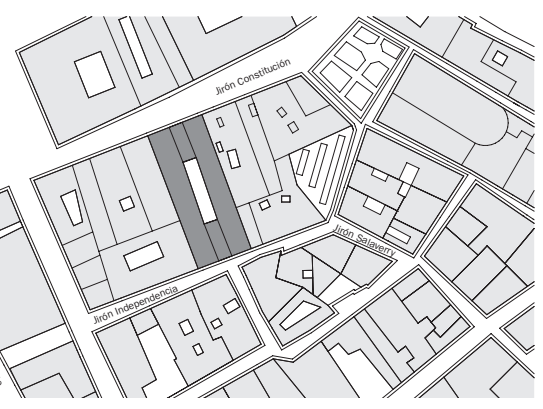

(1) ros y otros profesionales, a la vez que muchos de sus ambientes empezaron a quedar vacíos. Así, el edificio se hizo de una pátina de ruina contemporánea.

Si el pasaje Carmen representa la evocación tardía de una discreta, pero consistente puesta académica en la mejor tradición Beaux Arts, el pasaje Ronald se presenta como una manifestación enfática de modernidad, despliegue tecnológico y autoconciencia de inventar un nuevo territorio urbano. Sin embargo, en ambos casos la imitación de formas y estilos devino en un atajo ilusorio que velaba la distancia entre la metrópoli internacional y la periferia subdesarrollada, entre la autenticidad de lo moderno y el provincianismo

Las propuestas extensivas de demoli- $\mathbf{6}$ ción del patrimonio colonial, así como el ensanche de la red vial del centro y la construcción masiva de blocks formuladas por el Plan piloto de 1949, no lograron concretarse en toda su radicalidad. Sin embargo, el centro no volvería a ser el mismo espacio tras la concreción de algunos ensanches viales y la construcción de una y otra torre moderna ejecutada desde inicios de los cincuenta.

\section{MODERNIDAD Y EL PASAJE PRESCINDIBLE}

El Plan piloto de 1949, inspirado en las propuestas del Plan Voisin de Le Corbusier y los mandatos de los CIAM, propuso hacer desaparecer el centro histórico para inventar el paisaje arrollador de una nueva Lima moderna ${ }^{6}$. Paradójicamente, la central Plaza San Martín, inaugurada en 1921, se había convertido en el epicentro de la apuesta cultural moderna de Lima. Rodeada de librerías, cafés, comercios, los primeros grandes cines de Lima y toda aquella parafernalia dotada de modernidad tecnológica y visual, la plaza diseñada por Manuel Piqueras Cotolí se hizo espacio de referencia para Mario Vargas Llosa, José María Arguedas, Celia y Alicia Bustamante, Sebastián Salazar Bondy, Sérvulo Gutiérrez, Blanca Varela, Luis Miró Quesada y tantos otros exponentes de la vanguardia moderna limeña. Este es el momento y escenario en el que se inauguran los dos pasajes principales de la Lima moderna: la galería Boza (1956) y la galería Gallos-Mogollón (1959).

En 1956, a media cuadra de la Plaza San Martín se inaugura lo que en ese momento se autodefinió como la galería comercial más lujosa y moderna de Lima: la galería Boza, cuyo propietario fue el ingeniero Héctor Boza, vicepresidente del gobierno militar del general Manuel Odría (fig. 11). Se trata sin duda de un típico pasaje de corredor central que, con sus $115 \mathrm{~m}$ de largo y 28 de ancho, une en sentido transversal la cuadra ocho del jirón de La Unión, la calle comercial más importante de la época (fig. 12 y 13). De acuerdo a los tipos de pasajes identificados por Johann Geist, la Galería Boza se estructura espacialmente en base

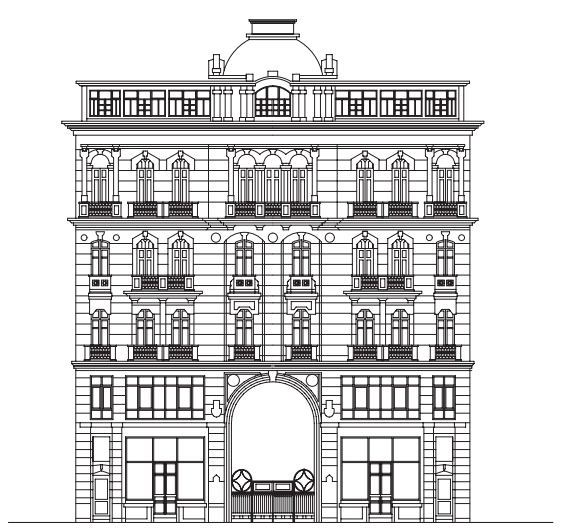

FIG 10

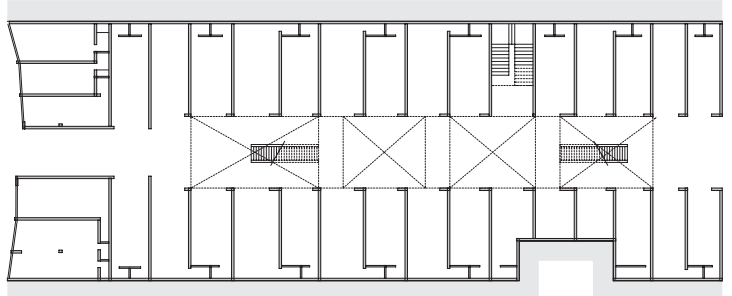

FIG 11

a "un corredor central recto-perpendicular de dos ingresos" (1989, p. 14). Al inaugurarse la galería inmediatamente se instalaron tiendas de lujo, oficinas de transnacionales de cine, librerías, restaurantes y cafés que no tardaron en hacerse célebres como el Café Galería y el Café Dominó. Este último se convirtió en uno de los lugares de encuentro predilectos de un sector de la vanguardia cultural y artística de la Lima de los cincuenta y sesenta. Hoy, la galería casi vacía en el segundo piso, intenta sobrevivir con el funcionamiento de tiendas deslucidas y un comercio precarizado. Los dos cafés históricos han desaparecido y la arquitectura empieza a perder sus atributos originales. 


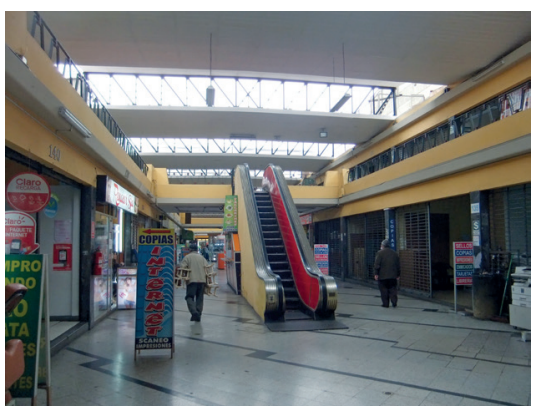

FIG 12

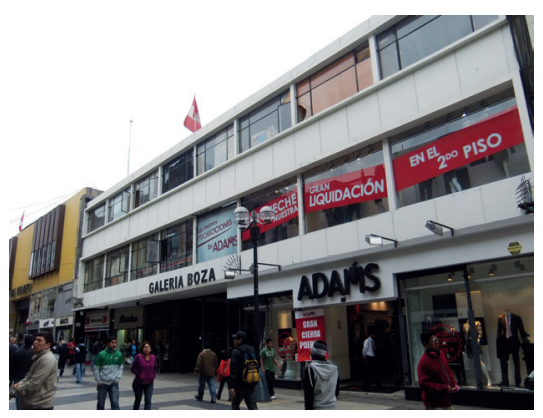

FIG 13

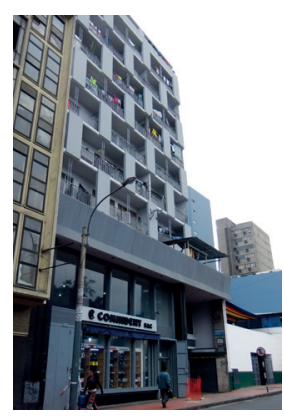

FIG 14

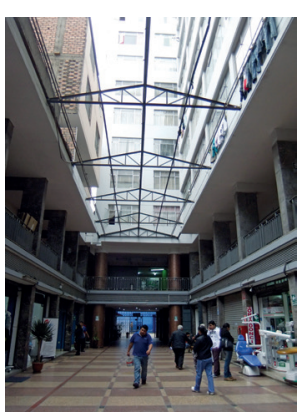

FIG 15

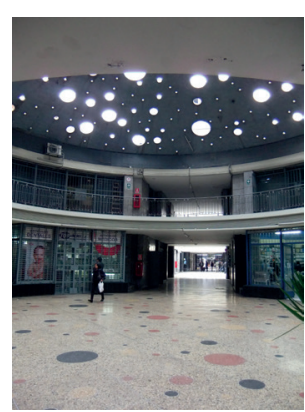

FIG 16

\section{FIG 12 Y FIG 13}

Fotografías: Wiley Ludeña Urquizo. Photography: Wiley Ludeña Urquizo.

\section{FIG 14 A FIG 18}

Galería Gallos-Mogollón, Centro Histórico de Lima, Perú, 1959.

Gallos-Mogollón Gallery. Historical center of Lima, Perú, 1959.

\section{FIG 14 A FIG 16}

Fotografías: Wiley Ludeña Urquizo. Photography: Wiley Ludeña Urquizo.

\section{FIG 17}

Planta primer nivel. E. 1: 1.000. Levantamiento: Diana Torres Obregón. First floor plan. Scale 1: 1.000. Survey: Diana Torres Obregón.

FIG 18

Corte Iongitudinal. E. 1: 1.000 Levantamiento: Diana Torres Obregón. Long section. Scale 1: 1.000 Survey: Diana Torres Obregón.

El barrio comercial de Gamarra se en- 7 cuentra ubicado en el tradicional distrito central de La Victoria. Ocupa una extensión variable de más de 63 manzanas en medio

del infierno urbano que aún representa el mítico barrio comercial de La Parada. Hoy, con un movimiento de más de 600 millones de dólares al año, este emporio de cientos de microempresas simboliza el emprendimiento popular y la pujanza del llamado capitalismo popular. Gamarra es un fragmento de barrio comercial informal que ha desarrollado sus propias capacidades de inserción global en medio de un mercado cada vez más adverso. Como sostiene Miriam Chion, es un caso ejemplar de la capacidad de un área marginal para

desarrollar los recursos institucionales apropiados que le han permitido conectarse con redes metropolitanas e internacionales (2002)

Los campos feriales se constituyeron $\mathbf{8}$ como una abigarrada trama de corredores angostos de no más de $2 \mathrm{~m}$ de ancho y cientos de minúsculos puestos de 2 a 3 $\mathrm{m}$ de ancho y fondo. Cubierto de toldos de plástico y otros materiales precarios, estos campos feriales se convirtieron en el símbolo manifiesto de una sociedad que,

como la peruana de la década de 1980 , estaba sumida en la peor crisis económica y política de su historia. Aquellos ubicados en el centro histórico fueron desmantelados y reubicados al borde del área central a mitad de la década de 1990, dando origen a los nuevos centros comerciales populares.
Con un mayor despliegue de arquitectura moderna, aspiraciones de mayor lujo y relativamente cerca de la galería Boza y del célebre Hotel Crillón, en 1959 se inaugura otro hito de modernidad arquitectónica en la ciudad: la galería Gallos-Mogollón (fig. 14). El pasaje se levanta en un lote de $111 \mathrm{~m}$ de largo, $35 \mathrm{~m}$ de ancho promedio y lados de trazo irregular y se estructura como un espacio longitudinal de doble altura. En tanto, la iluminación del pasaje tiene un registro cenital a través de un techo vidriado de dos aguas; se trata de un techo sencillo y sin mayor despliegue formal y técnico (figs. 15 y 16). Todo el espacio comercial se organiza en torno a un corredor de $10 \mathrm{~m}$ de ancho, recto y perpendicular a las calles de salida (figs. 17 y 18).

La galería Gallos-Mogollón nunca pudo convertirse en un espacio exclusivo de comercio y diversión del centro de la Lima moderna como pretendían sus propietarios. A partir de los años setenta, la crisis económica, el recambio social del centro de Lima, la expansión de la informalidad y precarización de la actividad comercial convirtieron la galería, tal como en otros casos, en otro espacio de comercio popular de baratijas y centro de mil oficios, cuando no de mercado popular de diversos usos.

Historia de una crisis anunciada: he ahí el destino final de estas dos galerías impregnadas de esplendor entrecortado y fracaso prematuro, pero también de un extraño desarraigo. Por un lado, las galerías Boza y Gallos-Mogollón se vieron abandonadas por la clientela tradicional a la que inicialmente estaban destinadas. Por otro lado, el nuevo sujeto social que empezó a ocupar el centro encontraba estos espacios de comercio insulsamente refinados y ajenos a sus condiciones de sobrevivencia. En cierto modo, la galería Boza y la Galería Gallos-Mogollón nacieron a destiempo o en el espacio equivocado, tanto que la historia de estas dos galerías resume en sentido literal y metafórico el estado de crisis permanente del centro histórico, sobre todo el registrado en las últimas tres décadas del siglo pasado.
CAPITALISMO POPULAR Y LA GALERÍA COMERCIAL. ¿VUELTA A LOS ORÍGENES?

El pasaje de esta primera década del siglo XXI se denomina galería comercial. Lo nuevo no es el nombre, sino el sujeto social que lo produce y usufructúa, y el sistema que lo promueve. Si el pasaje parisino nace como una expresión del nuevo lujo industrial, la galería comercial popular es la expresión consumada de una ciudad desindu strializada donde la pobreza aspira a diluirse bajo la estridencia de un lujo de utilería y luces de neón palidecido. Son la mejor expresión del desborde popular y de esa sociedad emergente que a diario inventa o trasgrede sus propias reglas de juego (Matos Mar, 2012). Esta ciudad emergente es la ciudad del reajuste neoliberal y neopopulista de los años noventa cuya única norma parece ser el más desembozado laissez-faire y laissez-passer. Se ha hecho estructuralmente informal y se ha transformado en una gigantesca feria con galerías en todos los intersticios de la ciudad.

La gran mayoría de estas galerías nacieron de manera espontánea e informal. Otras fueron objetos de diseño. Las hay de uno a dos niveles, así como edificios de más de diez pisos dedicados al comercio y otros usos productivos o servicios. En todos los casos estas estructuras conservan algo de las raíces originarias: por una parte el sentido fantasmagórico y de mundo autorreferencial del passage histórico y, por otro, la capacidad de embrujar, adormecer y convertir la mercancía en religión para transformar a los ciudadanos en clientes y consumidores casi sin voluntad propia.

Estas galerías venden mercancías, pero son a la vez mercancía misma que se mistifica como alucinógeno que debe hacer más soportable la vida de cientos de peatones limeños acosados por una ciudad de calles infernales y espacios desprovistos de auténtica vida pública. Algunos ejemplos son la galería Capón del Barrio chino de Lima, la galería Tarpuy o la galería Montevideo, por mencionar apenas algunas decenas de estas construcciones en los últimos años en el

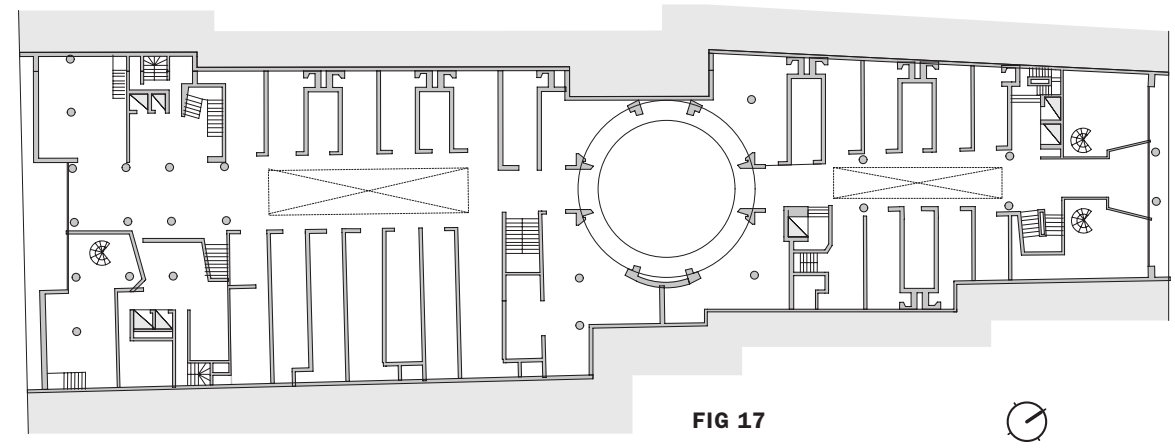




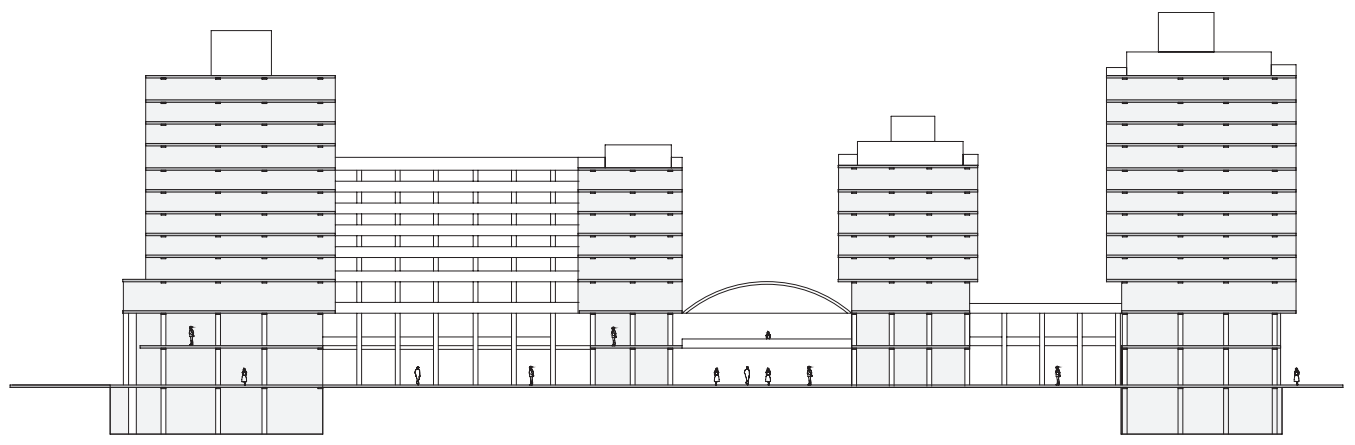

FIG 18

FIG 19 A FIG 26

Emporio Comercial Gamarra, Centro Histórico de Lima, Perú. Sin datos. Fotografías: Wiley Ludeña Urquizo. Gamarra Commercial Gallery, Historical center of Lima, Perú. No data. Photography: Wiley Ludeña Urquizo.

\section{FIG 27 A FIG 31}

Galerías Populares, Centro Histórico de Lima, Perú. Sin datos.

Popular galleries, Historical center of Lima, Perú. No data.

\section{FIG 27}

Galería Capón, planta de emplazamiento. E. 1: 7.500

Levantamiento: Diana Torres Obregón. Capón Gallery, site plan. Scale 1: 7.500. Survey: Diana Torres Obregón.

\section{FIG 28}

Galería Capón.

Fotografía: Diana Torres Obregón. Capón Gallery.

Photography: Diana Torres Obregón. área central de Lima y otras zonas de la capital. Estas galerías conservan el perfil de espacios estructurados por un pasaje de conexión externa a dos calles paralelas o transversales.

La expansión de la actividad comercial en la última década ha logrado caracterizarse como el boom inmobiliario de las galerías comerciales. El explosivo crecimiento de las galerías del emporio del comercio popular Gamarra es un dramático ejemplo7. De 40 galerías comerciales y seis mil unidades comerciales registradas en 1990, pasaron a ser 70 galerías y catorce mil unidades en 1997 (Ludeña, 2008). Hacia el 2011 estaban registradas 177 galerías comerciales y $14 \mathrm{mil}$ locales de negocio (Agencia Andina, 2011). Es una inmensa factoría o mercado popular en un espacio de 40 cuadras, saturado de miles de personas pugnando por vender, comprar o producir en medio de la basura y nuevas arquitecturas de estridente aspiración posmoderna. Las primeras galerías eran de dos a cinco pisos, en cambio las que existen hoy tienen más de diez. Un mundo aparte y alucinante: edificios mezcla de apretados talleres, zonas de venta de decenas de cubículos, restaurantes populares, ruido de redes telemáticas y ese típico color y olor del Perú profundo (figs. 19 a 26).

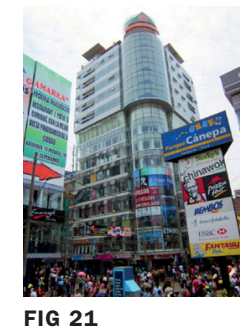

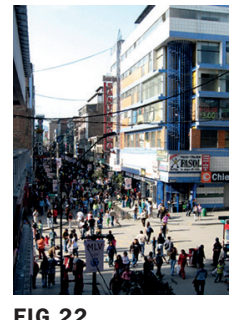

FIG 22

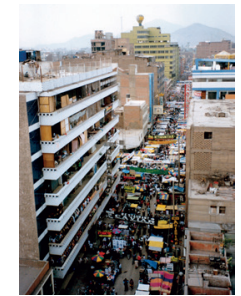

FIG 23
Junto a las nuevas galerías comerciales de diseño existe una tradición de galerías que se han constituido como una simple paráfrasis arquitectónica de aquellos campos feriales que se hicieron famosos durante la década de 1980. Los casos más conocidos fueron los campos feriales Polvos Azules y Amazonas, ubicados en el mismo centro histórico de Lima ${ }^{8}$. Las galerías comerciales que surgieron de esta operación se convirtieron casi en un auténtico modelo de optimización miserable del espacio comercial. Produjo ejemplos como la galería Centro Lima, la nueva galería comercial Polvos Azules (con los ambulantes reubicados del campo ferial del mismo nombre), la galería Las Malvinas (con parte de los veinte mil ambulantes reubicados de las calles del centro histórico) o la galería Polvos Rosados en Surco, entre otros.

Existe otro grupo de galerías comerciales que pueden caracterizarse como una constitución mixta de formato mediano y relativa formalización desde el diseño mismo. Se trata de galerías que, si bien registraban algunos antecedentes como aquellas que se construyeron en el jirón de La Unión, la galería Gran vía y la galería Vía Véneto, entre otras, empezaron a ser construidas a inicios de la década de 1990 y fueron repartidas en las principales zonas comerciales de la metrópoli. Sin embargo, la gran mayoría se encuentran ubicadas en torno al antiguo Mercado central, el Barrio chino y los jirones Paruro y Andahuaylas.

Desde el punto de vista morfológico las galerías populares registran una gran variedad de tipos, desde aquellas con corredores que conectan dos calles paralelas o transversales, hasta aquellas que poseen varios ingresos y una trama de conexión externa más compleja. También existen galerías de un corredor recto o en codos, así como en ángulo o segmentado con un patio central o algún tipo de espacio de convergencia. Existen galerías del tipo quinta que tienen múltiples entradas y del tipo de corredor central con edificios de dos o tres pisos a los costados. En este caso, el recorrido se estructura a partir de la presencia de un patio interior que le otorga sentido al conjunto.

Las galerías comerciales de hoy, si bien conservan elementos de referencia tipológicos que atañen a los pasajes de origen, han dejado de funcionar, entre otras cosas, como lugares de paseo y ocio reparador. Si antes los pasajes eran lugares de deslumbramiento por la variedad de productos y curiosidades provenientes de todas partes del mundo, las galerías populares son una despensa atosigada de baratijas y productos chinos e hindúes sin mayor sorpresa que la mala calidad de los mismos. No hay ninguna mitología detrás, salvo la deificación de la mercancía por la mercancía misma.
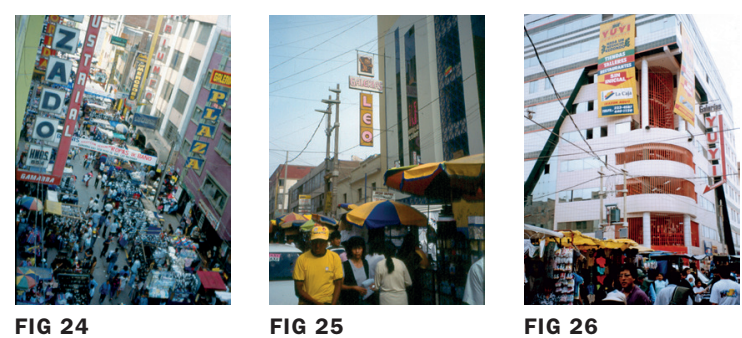

FIG 26

\section{TENSIONES ENTRE FORMALIDAD E} INFORMALIDAD COMERCIAL

La galería Capón se encuentra ubicada al interior del Barrio chino, uno de los sectores más antiguos y emblemáticos del centro histórico de Lima. El origen y éxito de la galería Capón no puede ser explicado sino es en el marco del extensivo plan de recuperación y renovación urbana del centro histórico, y del Barrio chino en particular, emprendido por la administración municipal desde mitad de la década de 1990 (figs. 27 y 28).

La galería se estructura como una edificación en forma de L para ocupar el interior de la manzana con un corredor central de un codo de $148 \mathrm{~m}$ de longitud, con anchos que oscilan entre los 11 y $18 \mathrm{~m}$. A ambos lados del corredor se ubican las tiendas y puestos en los que se comercia prácticamente todo, desde útiles escolares hasta ropa y enseres del hogar, pasando por 
Galería Capón, planta primer nivel. E. 1: 1.000. Levantamiento: Diana Torres obregón.

Capón gallery, first floor plan. Scale 1: 1.000. Survey: Diana Torres Obregón.

FIG 30

Galería Tarpuy.

Fotografía: Diana Torres Obregón.

Tarpuy Gallery.

Photography: Diana Torres Obregón.

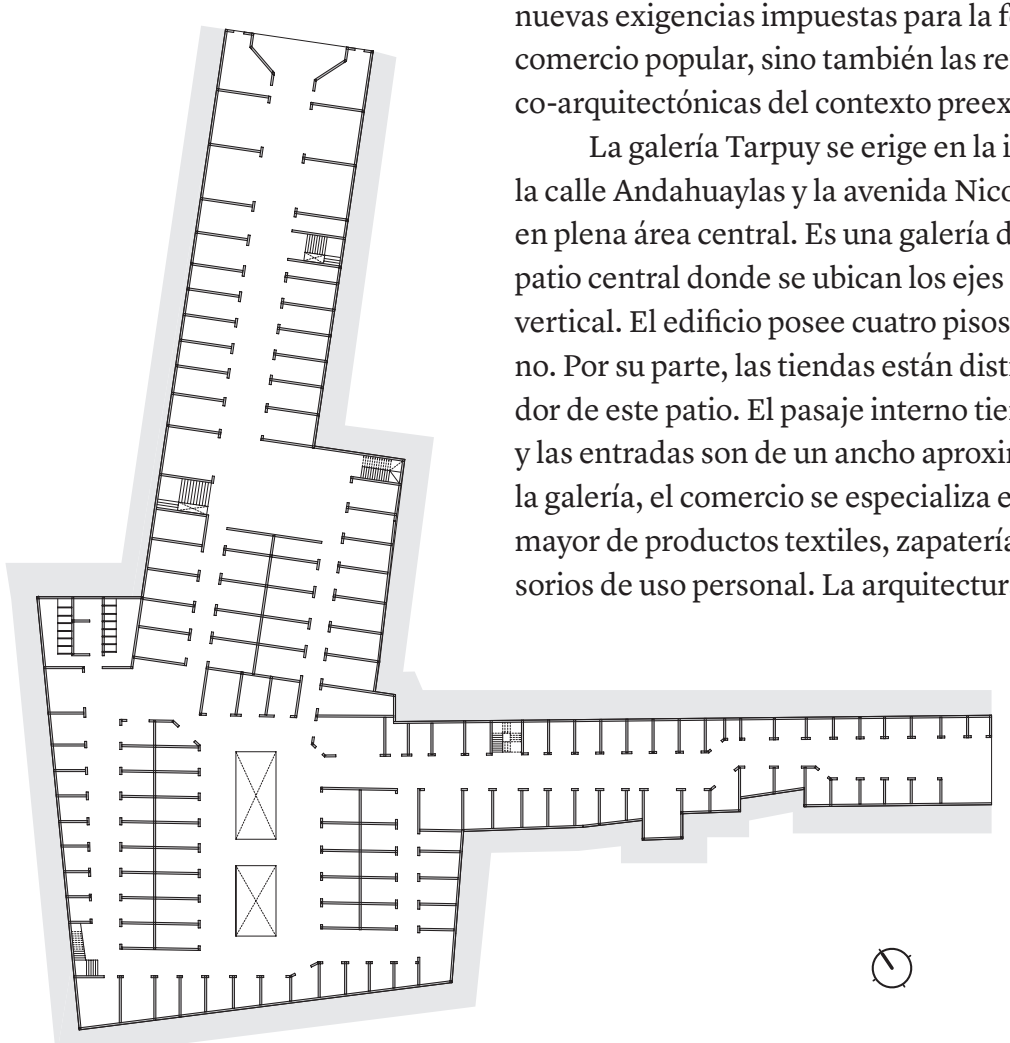

FIG 29

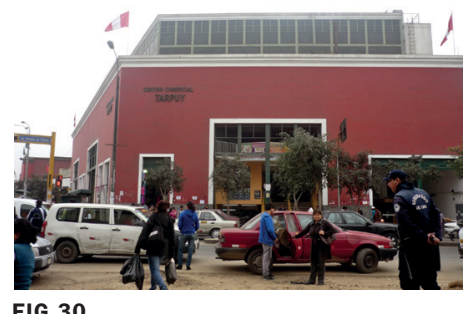

especias orientales, inciensos y adornos chinos. En el codo angular del corredor central se estructura un patio alargado que funciona como fuente de iluminación y ventilación natural de parte del conjunto. En el segundo piso se ofrecen los servicios de belleza y salud.

A diferencia de la galería Capón que representa una edificación de ordenamiento pragmático, funcional y sin pretensiones de orden arquitectónico, la galería Tarpuy expresa la voluntad de una nueva generación de comerciantes anteriormente informales, por proponer una arquitectura que recoja no solo las nuevas exigencias impuestas para la formalización del comercio popular, sino también las referencias históri-arquitectónicas del contexto preexistente.

La galería Tarpuy se erige en la intersección de a calle Andahuaylas y la avenida Nicolás de Piérola plena area central. Es una galería de conexión con patio central donde se ubican los ejes de circulación plantea como una reinterpretación histórica del lenguaje de las antiguas casas-patio coloniales de Lima. Aquí el efecto de mimesis se genera a través de una falsa fachada que simula un nivel de pertinencia contextual, sobre todo con el entorno urbano y la arquitectura de la iglesia de Santa Catalina (figs. 29 y 30).

Un tercer grupo de galerías comerciales populares lo constituye la serie de galerías del tipo canchón erigidas de manera precaria o provisional en un terreno amplio, de uso rústico o sin uso reconocido y no urbanizado. La mayoría de estas galerías consta de un piso con techo metálico y módulos prefabricados, también de metal. Los módulos de tiendas varían entre 5 y 8 $\mathrm{m}^{2}$ mientras que los pasadizos tienen un ancho que oscila entre 1 y 2,5 m. En conjuntos de galerías-canchón no es difícil evocar la estructura laberíntica de los bazares orientales. La galería Montevideo recoge plenamente los rasgos característicos de la galería del tipo canchón comercial; se ubica en la intersección de la avenida Montevideo y la calle Andahuaylas, ocupa un área de $1.700 \mathrm{~m}^{2} \mathrm{y}$ posee 160 tiendas, además de los servicios comunes básicos como un módulo de servicios higiénicos y un almacén (fig. 31). Como acontece con las otras galerías del mismo tipo, esta se destina al comercio especializado de ropa, calzado y accesorios de uso personal. A diferencia de las galerías formales del primer y segundo grupo, estas se constituyen en el reino de la venta de productos de imitación de marcas conocidas, denominados eufemísticamente como alternativos.

\section{PASAJES EN CONTROVERSIA.}

\section{A MODO DE CONCLUSIONES}

Si el passage es el símbolo urbano del capitalismo triunfante del siglo XIX, la galería comercial popular de inicios del siglo xxI es la expresión más descarnada del capitalismo salvaje, reeditado en países como el Perú, en clave neoliberal y neo populista para emplear las categorías de Kurt Weyland.

Los pasajes en Lima, los de antes y los de ahora nunca encarnaron una energía utópica ni fueron el mito de modernidad que debía prefigurar un nuevo mundo urbano. Nunca fueron el país de Jauja. Por el contrario, en muchos casos se convirtieron en un verdadero campo mortuorio de varios pisos con luces de casino, como en el dantesco incendio de la galería Mesa Redonda en el que fallecieron más de 300 personas dos días antes del año nuevo de 2002. Utopía al revés.

No obstante esta condición y otros atributos del mismo género ¿por qué las galerías populares continúan con una casi enloquecida expansión? ¿Cómo entender este fenómeno en una ciudad que como Lima carece precisamente de una tradición consistente de pasajes? ¿Cuáles son las razones de su éxito económico y social?.

Los pasajes surgieron en la capital peruana no precisamente porque el Perú se hubiera convertido en una pujante economía del capitalismo industrial, pero tampoco fracasaron porque no lo fueran. Igualmente, su expansión actual bajo el formato de galería comercial popular no implica la existencia consumada (o inexistencia) de un mundo urbano en ebullición global.

En realidad, la historia limeña de los pasajes se ha construido bajo la impronta de una condición básica. La persistencia de un imaginario urbano aún impregnado de nociones y valores de consumo ligados a la tradición árabe-ítalo-ibérica que, junto a una experiencia más pueblerina que metropolitana, convirtió al flâneur moderno en pura ficción antes que en personaje real. En este caso, esta persistencia tiene un doble significado: por un lado es el factor que retrasa el advenimiento de los pasajes, tal como sucedió en la Lima del periodo comprendido entre la primera mitad del siglo xIX y la primera mitad del siglo xx. Por otro lado, en sentido rigurosamente inverso, es el factor cultural que valida el boom neoliberal de las galerías populares: un auténtico homenaje tipológico a los pasajes y a la cultura del bazar re significado en América.

Salvo España y su tradición morisca, la presencia de los pasajes como formas de evocación al bazar oriental, resultaba a los ojos deslumbrados de Benjamin o Aragon una novedad y una deslumbrante revolución. Sin embargo, en un contexto como Lima con una extendida tradición de comercio impregnado del espíritu de bazar oriental, los pasajes concebidos a inicios del siglo $\mathrm{xx}$ podían parecer una inexplicable reiteración de lo existente. Por ello, en esta primera etapa los pasajes se revelaron apenas como una puesta en escena simulada tan artificial como las luces modernas de una ciudad estructuralmente anti 
FIG 31

Galería Tarpuy, planta primer nivel. E. 1: 750

Levantamiento: Diana Torres Obregón. Tarpuy Gallery, first floor plan. Scale 1: 750

Survey: Diana Torres Obregón.

FIG 32

Galeria Tarpuy.

Fotografía: Diana Torres Obregón.

Tarpuy Gallery.

Photography: Diana Torres Obregón.

FIG 33

Galería Montevideo, planta general. E. 1: 750

Levantamiento: Diana Torres Obregón. Montevideo Gallery, general plan. Scale 1: 750

Survey: Diana Torres Obregón.

\section{FIG 34 Y FIG 35}

Galería Montevideo.

Fotografías: Diana Torres Obregón. Montevideo Gallery.

Photography: Diana Torres Obregón.

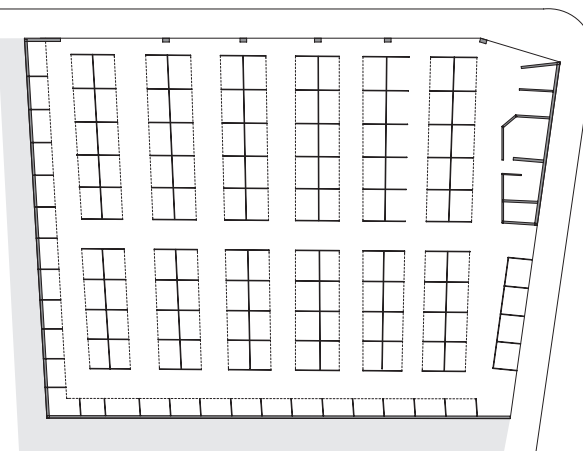

FIG 33

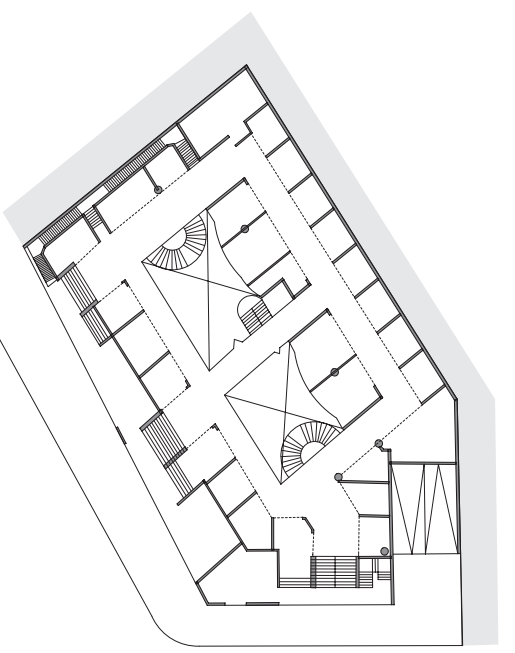

O

FIG 31

moderna. El bazar, con sus mercadillos y vendedores ambulantes en un patio o plazoleta, se impuso económica y socialmente a aquellos pocos pasajes construidos para una élite que nunca pudo ser tal ${ }^{9}$.

En una segunda etapa, la de la expansión actual de las galerías populares, el bazar deviene en una pulsión inconsciente que re significa cierta tradición para otorgarle legitimidad social y cultural a estos nuevos/viejos espacios de intermediación comercial, social y cultural. El bazar -o pasaje- no ha muerto: hoy se llama galería comercial. En este caso, a diferencia de las experiencias en el norte de Europa, no fue el pasaje el que evocó al bazar. En Lima, el bazar oriental arruinó y al mismo tiempo reinventó el pasaje: he ahí su repliegue inicial hasta la expansión desbordante actual.

Cada época construye su propia especificidad para transformar a los pasajes en experiencias cualitativamente distintas. El sujeto social del pasaje histórico europeo fue el flâneur. El sujeto social de los primeros pasajes limeños fue el sobreviviente de una sociedad sin élite social ni económica dispuesta a construir ciudad. Hoy, los pasajes posmodernos del capitalismo financiero y de la globalización neoliberal en el primer mundo se llenan de yuppies o banqueros sin capacidad de arrepentimiento. Las nuevas galerías comerciales de Lima se abarrotan al ritmo de lo que la sociología peruana identifica como la desterritorializada nueva clase media emergente llena de emprendimiento acrítico, despolitizada y culturalmente cínica.

Entre el pasaje como bazar preexistente, el pasaje de lujo y la galería comercial popular hay un hilo con-

FIG 34

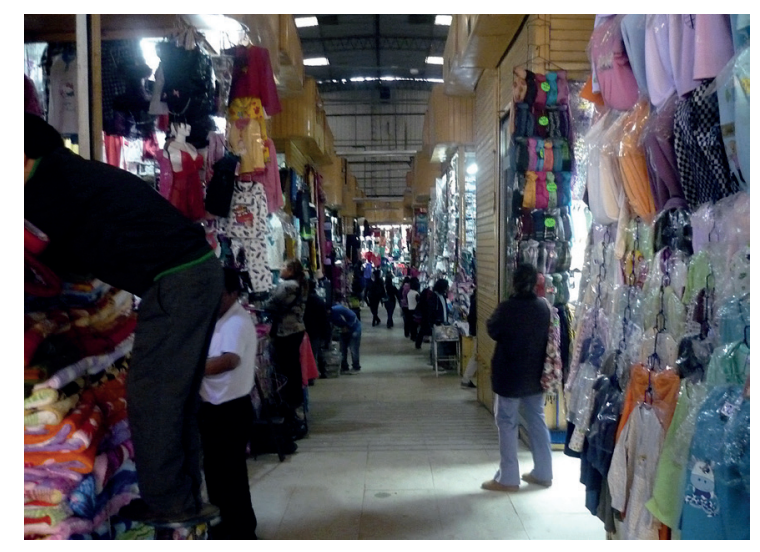

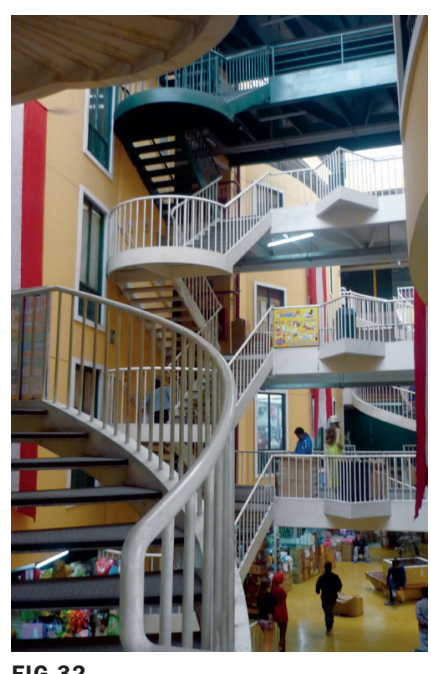

FIG 32

ductor, una especie de ADN compartido que les otorga el origen y sentido de pervivencia inevitable: la mercancía convertida en fetichismo activo, en objeto mistificado y mistificable. Si los pasajes históricos son aquellos templos donde el capitalismo industrial del siglo XIX deifica los valores de cambio y de uso de la mercancía, las galerías populares son templos en los cuales el capitalismo popular emergente se convierte en religión populista para producir exactamente el mismo ritual mistificador de la mercancía. Aquí, la inversión de los procesos y resultados no implica el rebatimiento de la tesis benjaminiana. Por el contrario, la valida de acuerdo a la relación sueño/ realidad y el reconocimiento de la función productiva y onírica de los pasajes y galerías comerciales.

Para Walter Benjamin los passages son la madre del movimiento DADA y el surrealismo (1982), pero cuando el autor de Das Passagen-Werk sostiene esto, no expresa nada que André Breton y el propio Louis Aragon no supieran cuando a fines de 1919 dejaron Montparnasse y Montmartre para refugiarse a soñar en un café del Passage de L'Opera. En el caso de Lima, lo extraño es que la pátina de lo onírico encarnado por los pasajes no aparece en sus versiones de inicio, sino más bien en su reinvención popular contemporánea. Es decir, las galerías comerciales de la Lima de hoy se han transformado en gigantescas y abigarradas naves de diseño tecno-andino-chicha-futurista con consumidores subsumidos por una lógica impregnada de sobrevivencia e informalidad emergente tan surrealista como el reino kitsch de la arquitectura de soporte. El passage ha muerto. jViva el pasaje!. ARQ

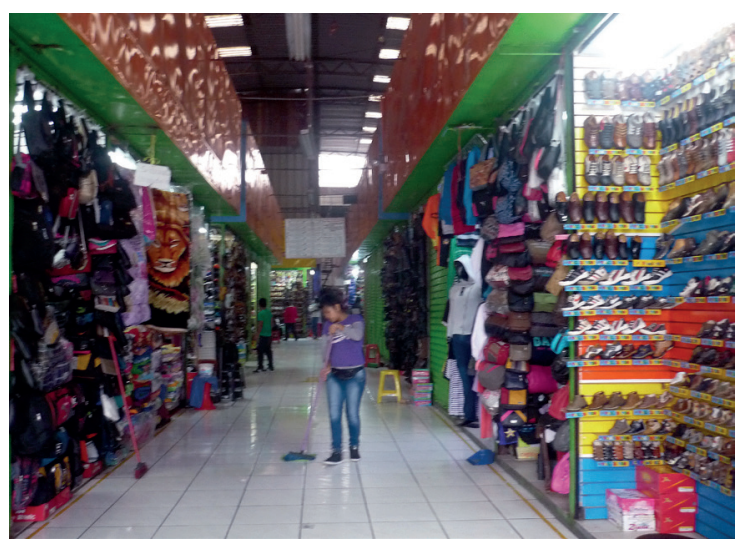

FIG 35
Debe reconocerse que en esta primera 9 etapa la razón climática igualmente pudo haber influido en la débil implantación de los pasajes en la Lima del novecientos. En ciudades sin lluvia y cambios extremos de clima, queda por lo menos en entredicho una de las principales razones de éxito del passage: ser un acogedor refugio temporal ante ese chubasco repentino o las inclemencias del tiempo en la ciudad (Benjamin, 1982). Los primeros pasajes en Lima no tuvieron más alternativa que reconocer que la ciudad del exterior seguía siendo el espacio privilegiado del consumo y la vitrina exultante de un flâneur tropical ansioso de ver y mostrarse. 


\section{REFERENCIAS BIBLIOGRÁFICAS}

AGENCIA ANDINA. "Gamarra produce S/.5,600 millones al año en confecciones". Andina, Agencia peruana de noticias. [En línea]. 25 de febrero de 2011, [fecha de consulta: 15 de julio de 2013]. Disponible en: <http:// www.andina.com.pe/agencia/noticia-gamarra-produceal-ano-s-5600-millones-confecciones-y-60-se-vende-elmercado-local-345046.aspx>

BENJAMIN, Walter. Libro de los pasajes. Rolf Tiedemann (ed.). Original c. 1927-1940 publicado en 1982. Madrid Ediciones Akal, 2004.

CHION, Miriam. "Dimensión metropolitana de la globalización: Lima a fines del siglo XX”. EURE, Revista Latinoamericana de Estudios Urbanos Regionales, XXVIII (85): 71-87, 2002

COELLO POHL, Tatiana. "Edith Martensen: Líneas hábiles que pintaron con amor al Perú”. Sociedad Amantes del País. [En línea]. 8 de marzo de 2009, [fecha de consulta: 15 de julio de 2013]. Disponible en: <http://amantesdelpais. wordpress.com/2009/03/08/edith-martensen-lineashabiles-que-pintaron-con-amor-al-peru/>

DÁVILA, Juan Manuel. "La casa Ronald. Relato de la Sra. Vivian Davies de McCoy". Callao Centro Histórico. [En línea]. 10 de marzo 2013, [fecha de consulta: 10 de julio de 2013]. Disponible en: <http://www. callaocentrohistorico.com/2013/03/la-casa-ronaldrelato-de-la-sra-vivian.html>

GARCÍA BRYCE, José. "Arquitectura en Lima 1800-1900". Amaru (3): 45-57, 1967.

GEIST, Johann. Le passage: un type architectural du XIXe siècle. Bruselas, Pierre Mardaga, 1989.

LUDEÑA URQUIZO, Wiley. Lima. Transformaciones urbanas y reestructuración económica. Periodo 1990-2005. Lima, Centro de Investigaciones de Arquitectura y la Ciudad CIAC, Pontificia Universidad Católica del Perú, 2008. MATOS MAR, José. Perú. Estado desbordado y sociedad nacional emergente. Lima, Universidad Ricardo Palma, 2012.

PARKER, David. The idea of the middle class: white - collar workers and Peruvian society 1990-1950. Pennsylvania, Pennsylvania State University Press, 1998.

WEYLAND, Kurt. "Neopopulismo y neoliberalismo en América Latina: afinidades inesperadas". PRETEXTOS, Revista del Área de Investigación Aplicada y Documentación de DESCO, (10): 7-43, septiembre de 1997

\section{BIBLIOGRAFÍA}

BASADRE, Jorge. Historia de la República del Perú 1822 1933. Lima, Editorial Universitaria, 1983. BENJAMIN, Walter. Discursos interrumpidos I. Filosofía del arte y de la historia. Rolf Tiedemann (ed.). Buenos Aires, Taurus, 1989.

LÓPEZ SORIA, José Ignacio. "Las lógicas de la modernidad". HUACA, Revista de la Facultad de Arquitectura, Urbanismo y Artes, Universidad Nacional de Ingeniería, (2): 4-9, abril de 1988.

LUDEÑA URQUIZO, Wiley. "Lima: poder, centro y centralidad. Del centro nativo al centro neoliberal". EURE, Revista Latinoamericana de Estudios Urbanos Regionales, XXVIII (83): 45-65, 2002

MARIÁTEGUI, José Carlos, 7 ensayos de interpretación de la realidad peruana. Lima, Empresa Editora Amauta, 1973.

MATOS MAR, José. Desborde popular y crisis del Estado. Veinte años después. Lima, Fondo Editorial del Congreso del Perú, 2004

MUÑOZ CABREJO, Fanni. Diversiones públicas en Lima 1890-1920: La experiencia de la modernidad. Lima, Red para el desarrollo de las Ciencias Sociales en el Perú, 2001.

ORTEGA, Julio. Cultura y Modernización en la Lima del
900. Lima, Centro de Estudios para el Desarrollo y la Participación CEDEP, 1986.

PINO, David. "La galería Mogollón". Lima la única. [En línea]. 16 de agosto de 2011, [fecha de consulta: 20 julio 2013]. Disponible en: < http://limalaunica.blogspot. com/2011/08/la-galeria-mogollon.html>

WILEY LUDEÑA | Arquitecto, Universidad Ricardo Palma 1978. Máster en Arquitectura, Universidad Nacional de Ingeniería, 1990. Doctor en Urbanismo, Technische Universität Hamburg-Harburg, 1996. Su trabajo como teórico e historiador comprende el estudio de los patrones de formación de los barrios en la ciudad peruana y latinoamericana del siglo XIX y la primera mitad del siglo XX, así como el análisis de los usos y transformación de los espacios públicos en las ciudades del Perú durante el periodo republicano. Desde 1980 ejerce la crítica periodística de arquitectura en diversos medios de la prensa peruana. Es fundador y director de la revista peruana de ciudad, urbanismo y paisaje ur[b] es. Ha sido director de la Maestría en Historia y crítica de la arquitectura y de la Maestría en Renovación urbana de la Universidad Nacional de Ingeniería. Actualmente se desempeña como profesor en la Universidad Nacional de Ingeniería y en la Pontificia Universidad Católica del Perú.

DIANA TORRES | Arquitecta, Universidad Nacional de Ingeniería, 2013. Coordinadora del Fondo Editorial de la Facultad de Arquitectura, Urbanismo y Artes de la Universidad Nacional de Ingeniería. Trabaja en la Dirección técnica de asentamientos humanos y adjudicación de tierras de la Municipalidad Metropolitana de Lima. Su ámbito de estudios se centra en la dinámica urbana de las áreas periféricas de la ciudad de Lima. Actualmente también se desempeña como profesora en la Pontificia Universidad Católica del Perú.

\section{FROM THE PASSAGE TO THE PEOPLE'S GALLERIES}

WILEY LUDEÑA | PROFESSOR, UNIVERSIDAD NACIONAL DE INGENIERÍA, LIMA, PERU.

DIANA TORRES | PROFESSOR, PONTIFICIA UNIVERSIDAD CATÓLICA DEL PERU, LIMA, PERU.

\section{OR FROM THE CRIOLLO LIBERALISM} TO THE NEO-LIBERAL CITY

While referring to Charles Fourier in Paris and the transfiguration of the passages into an authentic, idyllic phalanstery, Walter Benjamin evokes the country of Jauja, that medieval legend of a happy, self-sustaining country in a permanent state of ecstasy (1982). But the Peruvian Jauja is not completely the mythical Jauja, nor the passages of Lima that colossal human machinery that Fourier's dream suggested. ${ }^{1}$

In 1924 when the Pasaje Carmen opened (the first passage in Lima) the celebrated Passage des Panoramas, one of the first in Paris, turned 125 while the de L'Opera, the emblematic epicenter for Louis Aragon and the surrealists, was demolished after more than a century of existence. Ten years before this, in 1914, Buenos Aires already had streets with a splendor and size in some cases even more ostentatious than its European cousins such as the luxurious Bom Marché Gallery (1890) or the equally important Güemes Gallery (1913-1915).

Numerous galleries remain from this foundational period in some of the larger capitals in Latin America. Although the majority of them have already disappeared, some others are in the process of demolition, been converted to other uses or still keep its original function. In contrast, in some cities like Lima an explosive, vital and chaotic phenomenon has arisen in the last two decades: the expansion of a chich $a^{2}$ version of the $19^{\text {th }}$ century gallery in the format of the popular shopping gallery. Two moments and two contrasting frames of consumer architecture that unfold from the cycles of the same history and economic policy: that of $19^{\text {th }}$ century liberalism and the populist neo-liberalism of the $21^{\text {st }}$ century with their respective expressions in terms of city and architecture. ${ }^{3}$
The galleries are not a Parisian invention. Their precedents as a mode of commerce and building type are found in the oriental bazaar. As said by Walter Benjamin, the gallery, conceived as a temple to merchant capital, is the product of the bowels of the liberal capitalist city of the $19^{\text {th }}$ century ${ }^{4}$. In the same what that today, in the godforsaken swarm of the neoliberal city, the commercial galleries represent in many ways that commercial casino without a temple nor city that ceaselessly reproduces the voracity of that savage capitalism translated in aggressive neo-liberalism, skeptical of both the city and any form of citizenship.

If for $19^{\text {th }}$ century liberalism passages recreated a world as a complementary dream of the city with bewitched flaneurs and budding citizens, for neo-liberalism neo-populism the galleries exhaust this world, converting the city into a crumbling sub-product of the consumer imperative. If the liberal city of the $19^{\text {th }}$ century invented the passage and the post-liberal city of the $2 \mathrm{O}^{\text {th }}$ century proposed the civic center, the global command universalized the commercial galleries as a visceral expression of the most ominous and or positive dimensions of which Hernando de Soto calls popular capitalism.

Unlike other large capitals of the continent, Lima is not a city of passages emerged from a ubiquitous echo of the $19^{\text {th }}$ century and the European Belle Époque. But in the inverse sense and in contrast to these cities, the Peruvian capital is probably the most extreme example in Latin America of how the neo-liberal readjustment of the nineties has generated a limitless explosion of commercial galleries and other popular derivations of the old gallery. A paradox that is found in the middle of a single story in terms of architecture and economic policy: to less galleries of yesterday, more popular galleries today.
Or expressed in another way: less economic liberalism of yesterday, less galleries and to more populist neo-liberalism, more popular galleries.

The gallery, conceived as that iconic artifact of $19^{\text {th }}$ century capitalism arrived in Peru in the second decade of the $2 \mathrm{O}^{\text {th }}$ century as a symbol of modernity still elusive in the middle of a structurally anti-modern city. Therefore, Lima has been for this dream machine like that farmer of Louis Aragon in Le paysan de Paris (1926), a provincial city that glares at the new; a city that doesn't invent the galleries itself, but adopts it to marvel and dramatize the experience of another life.

It is this distance and essential uprooting that surrounds the origin and history of the Lima galleries. It marks a history of banal fixation for the new but at the same time produces a social indifference also of sudden modernity and premature obsolesce. An example of this is the Carmen Gallery, the first built gallery in Lima that today survives with precarious stores and kiosks selling postcards and trinkets. In the same way, the Ronald Gallery (1929) built in the port of Callao, an authentic tribute to the best architectural tradition of the European galleries, never truly functioned as such and today is almost a strange ghost that tries to revive from time to time. The same happens with the two modern galleries in Lima: the Boza Gallery (1956) and the Gallos-Mogollón Gallery (1959).

Perhaps in this contradiction and complex architectonic, urban and cultural notation resides the magic of this series of first and only galleries built in the oligarchic and conservative Lima of the $2 \mathrm{O}^{\text {th }}$ century. Each one with a history that does not cease to be equally surrealist like the intensive and colorful daily use that today is housed within a degraded and spooky 
landscape. But this is a story that began to change dramatically two decades ago with the drastic neo-liberal and neo-populist readjustment promoted by the government of Alberto Fujimori (1990-200o). Although before Fujimori a kind of invasion would be produced of streets and market grounds in the center of Lima in its most miserable and precarious expression, at the end of the nineties an authentic explosion and seedy refinement would occur from which in Peru are called the popular commercial galleries.

This phenomenon is the expression of the new cycle of economic expansion that Peru experiences after the structural readjustment and the subsequent return to primary forms of savage capitalism. Here are the dozens of new commercial galleries erected in the las few years in the most well known commercial neighborhoods in Lima: the central market zone and the Gamarra, the Malvinas and Abancay Avenue commercial neighborhoods and more. The commercial galleries today are the galleries of yesterday. The recreate the typological fundamentals of the bazaar and the $19^{\text {th }}$ century gallery, but they are no more than a sacred temple of mercantile capitalism. They are the new shril and soporific commercial casinos designed at the same time by the late global capitalism and the consensus of Washington for the peripheral countries and the thousands of poor consumers.

\section{LOS PASSAGES DEL LIBERALISMO CRIOLLO}

The first Lima gallery, the Carmen Gallery, opened its doors in 1924 during the first centennial independence celebrations and the Ayachucho battle day. The event formed part of the national holidays activities. The Ronald Gallery, the second in the decade, opened in 1929 almost in the aftermath of the Augusto B. Leguía government (1919-1930), self-proclaimed as the government of the New Country.

How is it possible that a building of consummate Beaux Arts orthodoxy like the Carmen Gallery come out of a Lima that aspired to leave behind any sign of $19^{\text {th }}$ century architecture and conservative historical eclecticism in that time? And second, how can is be understood that a building like the Ronald Gallery was built as a futurist enclave when the prosperity of Leguia was a thing of the past and nothing justified such excess?

In reality, the galleries were in the urban imagination of some Limeños illustrated with an experience of European live during the last quarter of the $19^{\text {th }}$ century. That the first case has been built almost half a century later is fundamentally due to two factors: the period of economic depression that the Pacific War (1879-1883) produced and the absence of structural transformations in the city. Those projects would recently begin to be produced at the beginning of the $2 \mathrm{O}^{\text {th }}$ century upon resuming the Haussmann-inspired modernizing project of restructuring Lima by José Balta, Nicolás de Piérola and Luis Sada between 1868 and 1872 .

Unlike the European experience in which the galleries preceded the large department stores, in Lima the opposite occurred. This fact would decisively influence the destiny of the galleries and their weak institutionalization such as their eventual commercial economic failure. In 1917, when the German immigrant, Augusto Fernando Oechsle opened the first modern department store along the Plaza Mayor, the galleries of the future had an uncertain future. The first victim: the Carmen Gallery located less than $200 \mathrm{~m}$ from the Casa Oechsle.

In a society with an impoverished middle class and a conservative, closed and distant oligarchy full of huachafos and huachafitas, ${ }^{5}$ as noted by David S. Parker, the public or semi public spaces like cafes, restaurants and galleries never achieved a degree of empathy and social legitimacy with high society. The spaces privileged by the elite made up a self-referential world of clubs or closed businesses similarly to the Casa Oechsle, protected from the social infiltrators (Parker, 1998).
THE CARMEN GALLERY AND THE LATE EVOCATION OF THE ARISTOCRATIC REPUBLIC

The Carmen Gallery makes up one of the singular components of a group of three structured buildings of the Casa de Correos y Telégrafos (1897). It is found in the middle of a block located in a strategic zone in the center of Lima: next to the Government Palace and close to a corner of the Plaza Mayor. The gallery, $115 \mathrm{~m}$ long and 6 $m$ wide, links the streets Camaná and La Unión (fig. 1). It was designed by the architect Raúl María Pereira, and was built in 1924 .

The gallery, in order of appearance is the third building in the group, was conceived as an integrated component with the academic architecture of Emilio Parzo and Máximo Doig, responsible for the design of the Casa de Correos y Telégrafos, considered one of the best examples of Beaux Arts architecture in Lima. With the intention of radiating a manifest monumentality with the front entrances, the architecture of the Carmen gallery, in its two plans, collects a compositional coherence to the typological fundamentals of a tradition perfectly decanted for that time (figs. 2 y 4 ).

A barrel vault with a glass roof covers the central corridor. This vault is converted into a structure of edges and particular structural development to cover the central patio, giving it a visible monumentality ${ }^{6}$. The steel structure of the roof marks the rhythm of a structural sequence composed from the basement of the galleries (figs. 3, 5 and 6). As characterized by José García Bryce, "the gallery architecture is structured around the neo-renaissance repertoire of the interior facades of the gallery and the baroque notation of the corbels, balustrades, column orders and pilasters" (García Bryce, 1967, p. 180). The entrances on both sides are indisputable representation elements of the gallery. Those are composed of an elaborate display of neo-classical, historical components endowed with a monumentality of a certain baroque drama. The doorways make sacred a space that is profane by nature.

The Carmen Gallery is not converted into a mythical meeting place or a symbol of a commercial or cultural time in the history of Lima. It functioned basically as an annex to the activities of the postal world. However, it also had commerce, restaurants or shops that at its best time -that of the Leguía period and the years after it- received part of the Lima elite and much more of the governmental bureaucracy.

\section{THE RONALD GALLERY OR THE LOST}

\section{DREAM OF THE NEW COUNTRY}

Construction began on the Ronald Gallery in 1923 and was opened in 1929, the same year of the crack of New York and only months before the Leguia government debacle. Maybe this explains its opaque destiny: it never achieved the splendor dreamed of by its owner. The concept and design correspond decidedly to the personal will of Guillermo Ronald, who wanted to reproduce in Callao the format and aesthetic of the more refined arcades he had frequented in Europe. Nicolaus Babinski, Austro-Hungarian architect living in Lima and the British engineer, Bunting, participated in the design and construction (Coello Pohl, 2009; Dávila, 2013).

The building crosses an irregular block, one side of which faces the main church square. The passage, $6 \mathrm{~m}$ wide and 55 long, links Constitución and Independencia streets. The lot and building that occupy it are $30 \mathrm{~m}$ width (fig. 7). The resulting volume is an enormous artifact of 6 floors whose proportions are superlative in the urban context, which has an average of two floors. Together with the Casa Wiese (1922) and the Gildemeister building (1928), both 6 floors and located in the center of Lima, the Ronald Gallery is another of Lima's first skyscrapers. It held more than 90 spaces for offices and businesses as well as 12 apartments that housed prominent families from the English colony of the port. On the fifth floor there was a celebrated English bar known as Twin Deck. Its luxurious elevators were the first of Callao.
Along with this, the first floor gallery manifests solemnity and grand spatiality. This is reinforced with the marble floors and stairs, wooden panels, friezes and iconic capitals, medallions and other details designed with the principles of a certain neo-classicism evoked in a modern key. The double-height commercial corridor has a glass ceiling. Here the overhead lighting acquires an effect due to the elaborately artistic glass (fig. 8). In the Ronald Gallery the idea of the gallery building is made evident from the exterior. The building is the gallery and this in its interior structure expresses the will of the building as architecture and as a world of multiple activities. That is, a building city. Lifting its face to the Callao port, the building is transformed not only into a landmark but a kind of mercantile lighthouse that dominated the profile of the port and marks its morphological configuration (figs. 9 and 10).

It his best and short time, the Ronald Gallery was the epicenter of the most modern and European of the Callao port. It was the meeting place of businessmen and sea officers and numerous European families that lived in La Punta neighborhood or in the apartments of the upper floors. With time the offices began to be occupied by different administrative, sports or maritime institutions and many spaces began to be left vacant. And so the building became a contemporary ruin.

If the Carmen gallery represents the evocation of a discreet but consistent proposal of the Beaux Arts tradition, the Ronald gallery is presented as an emphatic manifestation of modernity, and consciousness to invent a new urban territory. However, in both cases the imitation of forms and styles became an illusory shortcut that veiled the distance between the international cities and the under-developed periphery, between the authenticity of the modern and the rhetorical provincialism, between critical universality and conservative cosmopolitanism.

\section{MODERNITY AND THE DISPENSABLE GALLERY}

The Pilot Plan of 1949, inspired by the Le Corbusier's Plan Voisin and the mandates of the CIAM, proposed destroying the historic center to invent an overwhelming landscape of a new modern Lima7 ${ }^{7}$ Paradoxically, the central Plaza San Martín, opened in 1921, had been converted in the epicenter of modern culture in Lima. Surrounded by bookshops, cafes, shops, the first cinemas and all the paraphernalia of technological and visual modernity, the plaza designed by Manuel Piqueras Cotolí was a reference for Mario Vargas Llosa, José María Arguedas, Celia and Alicia Bustamante, Sebastián Salazar Bondy, Sérvulo Gutiérrez, Blanca Varela, Luis Miró Quesada and so many other exponents of modern avant-garde in Lima. This is the moment in which the two main galleries of modern Lima were opened: Boza Gallery (1956) and Gallos-Mogollón Gallery (1959).

In 1956, half a block away from the Plaza San Martín, they opened what in that moment was called the most luxurious and modern gallery of Lima: the Boza Gallery, whose owner was the engineer, Héctor Boza, vice president of the military government of General Manuel Odría (fig. 11). Without a doubt, it deals with a typical central corridor gallery that, with its $115 \mathrm{~m}$ of length and $28 \mathrm{~m}$ of width, united the $8^{\text {th }}$ block of La Unión crosswise, the most important commercial street of the time (fig. 12 and 13). According to the kinds of galleries identified by Johann Geist, Boza gallery is structured spatially around a "central recto-perpendicular corridor with two entrances" (1989, p. 14). Upon opening the gallery was immediately filled with luxury stores, trans-national offices of cinema, bookshops, restaurants and cafes that in no time became noted such as the Café Galería and the Café Dominó. The latter became one of the favorite meeting places of a sector of cultural and artistic avant-garde of Lima in the fifties and sixties. Today, the gallery is almost vacant, trying to survive with dingy shops and precarious business. The two historical cafes have disappeared and the architecture has begun to lose its original attributes. 
With an increased appearance of modern architecture, aspirations of luxury and close to the Boza Gallery and the celebrated Hotel Crillón, in 1959 another landmark of modern architecture in the city was opened: the GallosMogollón Gallery (fig. 14). The gallery stands on a lot 111 $\mathrm{m}$ long, $35 \mathrm{~m}$ wide with an irregular outline and is structured as a long double-height space. Meanwhile, a gabled glass roof lights the gallery; it is a simple roof without formal or technical complexity (figs. 15 and 16). All the commercial space is organized around a $10 \mathrm{~m}$ wide straight corridor perpendicular to the streets (figs. 17 and 18).

The Gallos-Mogollón Gallery never became an exclusive commercial and leisure space in the center of modern Lima as its owners hoped. Starting in the seventies, due the economic crisis and downtown Lima social transformations, the expansion of the informality of the commercial activity transformed the gallery, like in other cases, into popular trinket shops.

Story of a crisis foretold: this is the fate of those two galleries impregnated with splendor and premature failure but also of a strange upheaval. On one hand, the traditional clientele for whom they were originally built abandoned the Boza and Gallos-Mogollón galleries. On the other hand, the new social subject that began to occupy the city's central areas found these spaces to be overly refined and far from their survival conditions. In a certain way, the Boza and Gallos-Mogollón Galleries born at the wrong time or the wrong place while the story of these two galleries summarizes in a literal and metaphorical sense the state of permanent crisis in Lima's historical quarter, especially during the last three decades of the last century.

\section{POPULAR CAPITALISM AND THE COMMERCIAL} GALLERY. A RETURN TO ORIGENS?

The gallery of this first decade of the $21^{\text {st }}$ century is called the commercial gallery. The name is not new, but the social subject that produces it, uses it and the system that promotes it. If the Parisian gallery was born as an expression of the new industrial luxury, the popular commercial gallery is the consummate expression of a de-industrialized city where poverty aspires to dilute itself with the stridency of a luxury or props and pale neon lights. They are the best expression of the popular overflow and emerging society that invents or breaks its own rules (Matos Mar, 2012). This emerging city is the city of the neo-liberal, neo-populist readjustment of the nineties whose only rule is an undisguised laissez-faire and laissez-passer. It has become structurally informal and has been transformed into a giant market with galleries at every intersection of the city.

The vast majority of these galleries were born spontaneously. Others were objects of design. They are one floor or two, or ten-story buildings dedicated to commerce and other productive uses or services. In all cases these structures conserve something of their roots: the spooky and self-referential world of the historical passage and the capacity to bewitch, numb and convert consumerism into a religion to transform citizens into consumers without a will of their own.

These galleries sell merchandise, but are at the same time merchandise themselves that mystifies like a hallucinogen that makes life more bearable for the hundreds of pedestrians harassed by a city of crowded streets and spaces devoid of authentic public life. Some examples are the Capón Gallery in Lima's Chinatown, the Tarpuy Gallery or the Montevideo Gallery to mention a few of the dozens of these constructions built within the last years in the central area of Lima and other areas in the capital. These galleries conserve the profile of spaces structures by an external connecting corridor.

The expansion of commercial activity in the last decade has been characterized as a real estate boom of commercial galleries. The explosive growth of galleries of the popular trading emporium Gamarra is a dramatic example ${ }^{8}$. From 40 commercial galleries and six thou- sand commercial units registered in 1990, it became 70 commercial galleries and 14,ooo stores (Agencia Andina, 2011). It is a huge factory or popular market in a space of 4o blocks, saturated with thousands of people selling and buying among the trash and new architecture of strident postmodern aspiration. The first galleries were two to five stories whereas today they have more than ten. A world of its own: buildings mix narrow workshops, sale areas with dozens of cubicles, fast food restaurants, telemetric network noise and the typical color and ambience of deep Peru (figs. 19 to 26).

Along with the new design arcades there is a tradition that has been established as a simple architectural paraphrase of those markets that were made famous during the eighties. The better-known cases were the Polvos Azules and Amazonas markets located in the historical center of Lima9. The commercial galleries that arose from this operation were converted into a miserable optimization model of the commercial space. It produced examples such as the Centro Lima Gallery, the new Polvos Azules commercial gallery (with the vendors from the market of the same name relocated) Las Malvinas Gallery (with part of the twenty thousand vendors relocated from the streets of the historic center) or the Polvos Rosados Gallery in Surco, among others.

There exists another group of commercial galleries that can be characterized as a medium format mixed constitution and relative formalization from the design itself. They are galleries that register some background like those that were built in La Unión, the Gran Via Gallery and the Via Veneto Gallery among others that began to be built at the beginning of the nineties and were distributed through the main commercial zones of the city. However, the large majority is found located around the old Central Market, Chinatown and the Paruro and Angahuayalas neighborhoods.

From the morphological point of view, the popular galleries record a large variety of types from which they connect parallel or transversal streets to those that possess various entrances and a complex web of external connections. There are also galleries of a straight corridor or with bends or segmented, with a central patio or some kind of convergent space. There are galleries of a fifth type that have multiple entries and of the kind of central corridor with buildings of two or three floors on the sides. In this case, the path is structured around the presence of an interior patio that gives meaning to the whole.

The commercial galleries of today, while conserving the typological reference elements regarding the original galleries have ceased to function as leisurely places to stay. If before the galleries were places of awe from the variety of products and curiosities from all over the world, the popular galleries are a dispensary of cheap Chinese and Indian products with nothing more surprising than their poor quality. There is no mythology behind them besides the deification of the merchandise for the sake of merchandise.

\section{TENSIONS BETWEEN COMMERCIAL FORMALITY AND} INFORMALITY

The Capón Gallery is located to the interior of

Chinatown, one of the oldest and emblematic areas of the historic center. The origin and success of the Capón Gallery cannot be explained but is part of the extensive recovery plan of urban renovation of the historical center undertaken by the municipal administration since the mid-nineties (figs. 27 and 28).

The gallery is organized in the form of an L occupying the interior of a block with a central bent corridor $148 \mathrm{~m}$ long whose width oscillates between 11 and $18 \mathrm{~m}$. There are stores on both sides of the corridor where almost everything is sold, from school supplies to clothing and household goods, oriental spices, incense and Chinese decorations. In the central corridor there is a long patio that functions as a source of light and natural ventilation. On the second floor there are health and beauty services.
Unlike the Capón Gallery that represents a pragmatic, functional and unpretentious architectural system, the Tarpuy gallery expresses the will of a new generation of previously informal venders to propose an architecture that no only responds to the new demands imposed by the formalization of popular commerce but also the historical architecture references of the pre-existing context.

The Tarpuy Gallery stands at the intersection of Andahuaylas street and Nicolás de Piérola Avenue, in the heart of downtown Lima. It is a connection gallery with a central patio where there are two vertical circulation axes. The building has four floors and a semi-basement. The stores are distributed around this patio. The internal gallery is $39 \mathrm{~m}$ long and the entrances are approximately 5 $\mathrm{m}$ wide. In the gallery, the stores specialize in bulk sales of textiles, shoes and accessories for personal use. The architecture of the gallery is planned as a historical reinterpretation of the old colonial patio-houses of Lima. Here there is a false facade that simulates a level of contextual belonging, above all with the urban surroundings and the architecture of the Santa Catalina church (figs. 29 and 30).

A third group of popular galleries makes up a series of galleries of a kind of field built precariously or provisionally on an undeveloped large site. The majority of these galleries have a single story with a metal roof and prefabricated modules, also metal. The modules of the stores vary between 5 and $8 \mathrm{~m}^{2}$ while the pathways vary between 1 and $2.5 \mathrm{~m}$ wide. In these warehouse galleries it is not difficult to evoke the mazelike structure of the oriental bazaars. The Montevideo Gallery clearly reflects the characteristics of the warehouse-gallery; located on the intersection of Montevideo Avenue and Andahuaylas street, it occupies an area of $1.700 \mathrm{~m}^{2}$ and has 160 stores as well as basic common services such as bathrooms and storage (fig. 31). As it happens with other galleries of the same type, it specializes in clothing, shoes, and personal accessories. Unlike the formal galleries of the first two groups, these constitute the world of imitations of known brands, known euphemistically as alternatives.

\section{GALLERIES IN CONTRAVERSY. CONCLUSIONS.}

If the passage is the urban symbol of triumphant capitalism of the $19^{\text {th }}$ century, the popular commercial gallery of the beginnings of the $21^{\text {st }}$ century is the starkest expression of savage capitalism, reedited in countries like Peru, in a neo-liberal and neo-populist key to employ the categories of Kurt Weyland.

The galleries in Lima, those from before and those from now never embodied a Utopian energy nor were the myth of modernity that would prefigure a new urban world. They never were the country of Jauja. On the contrary, in many cases they became a multi-story mortuary adorned with casino lights, like the Mesa Redonda Gallery fire in which more than 300 people lost their lives before New Years in 2002. Utopia backwards.

However given this condition and other similar attributes why do the popular galleries continue their crazy expansion? How can we understand this phenomenon in a city like Lima, which lacks a consistent tradition of galleries? What are the reasons for their social and economic success?

The galleries didn't emerge in the Peruvian capital because Peru had become a thriving economy of industrial capitalism, but they didn't failed because they were not. Equally, their current expansion under the popular commercial gallery format does not imply the consummate existence (of absence) of an urban world at a global boiling point.

Currently, the history of galleries of in Lima has been built under the idea of a basic condition. The persistence of an urban fantasy still impregnated with notions and values of consumption linked to the ArabItalian-Spanish tradition that, with a more parochial than metropolitan experience, converted the modern flâneur into fiction before a real character. In this case, this persistence has a double meaning: on one hand it is 
the factor delaying the advent of the galleries as it happened in Lima between the first half of the $19^{\text {th }}$ century and the first half of the $2 \mathrm{O}^{\text {th }}$ century period. On the other hand, in a rigorously reverse sense, it is the cultural figure that validates the neoliberal boom of the popular galleries: an authentic typological tribute to the galleries and bazaar culture reinvented in America.

Except for Spain and its Moorish tradition, the presence of the galleries evoking the oriental bazaar, dazzled the eyes of Benjamin or Aragon as a novelty and a sparkling revolution. However, in a context such as Lima with an extended tradition of trade impregnated with the spirit of the oriental bazaar, the galleries conceived at the beginning of the $2 \mathrm{O}^{\text {th }}$ century could appear as an inexplicable reiteration of the existing. Therefore, in this first stage the galleries are barely revealed as a scene simulated as artificially as the modern lights of a structurally anti-modern city. The bazaar, with its markets and street vendors in a patio or small plaza, was socially and economically imposed to those small galleries built for an elite that would never be..$^{10}$

At a second stage, the current expansion of the popular galleries, the bazaar becomes an unconscious drive that redefines a certain tradition to give social and culturally legitimacy to these new/old spaces of commercial, social and cultural inter-mediation. The bazaar (or gallery) has not died: today it is called a commercial gallery. In this case, unlike the experiences in the north of Europe, it was not the gallery that evoked the bazaar. In Lima, the oriental bazaar ruined and yet at the same time reinvented the gallery: behold the initial withdrawal to the current overwhelming expansion.

Every era builds its own specificity to transform the galleries in qualitatively different experiences. The social subject of the historical European gallery was the flâneur. The social subject of the first Lima galleries was the survivor of a society with neither a social nor economic elite available to build a city. Today, the post-modern galleries of the financial capitalism and the neoliberal globalization in the first world are filled with yuppies or bankers without capacity to repent. The new commercial galleries of Lima are filled to the rhythm of what Peruvian sociology identifies as the de-territorialized new emerging middle class full uncritical, depoliticized and culturally cynical enterprise.

Between the gallery as a pre-existing bazaar, the luxury gallery and the popular commercial gallery there is a common thread, a kind of shared DNA that gives them the origin and meaning of inevitable survival: the merchandise converted into active fetishism, into a mystified object. If the historical galleries are those temples where the industrial capitalism of the $19^{\text {th }}$ century deifies the exchange values and the use of goods, popular galleries are temples in which the emerging popular capitalism is converted into populist religion to produce the same mystifying ritual of merchandise. Here, the reversal of the processes and results does not imply the feathering of Benjamin's thesis. Quite the contrary, it validates his ideas considering the links between dream and reality and the recognition of the productive and oneiric functions of the passages and commercial galleries.

For Walter Benjamin the passages are the mother of the DADA movement and surrealism (1982), but when the author of Das Passagen-Werk sustains this, he expresses nothing that André Breton and Louis Aragon themselves did not know when, at the end of 1919, they left Montparnasse and Montmartre to hideaway and dream in a cafe in the Passage de L'Opera. In the case of Lima, the strange thing is that the patina of dreams embodied by the galleries does not appear in the initial versions, but in its contemporary popular reinvention. That is, the commercial galleries of Lima today have been transformed in gigantic and variegated techno-Andean-futuristic design ships with consumers subsumed by a logic that blends emerging survival and informality, as surrealist as the kitsch kingdom of the architecture of support. The passage has died. Long live the gallery!

\section{Notes}

1 Although descriptive and elemental in its formulation, the definintion of the passage created by the Guia Ilustrada de París (1852) proves accurate: "they are large glass-roofed galleries, encased in marble that cross entire buildings whose owners have joined together for these speculations. Both sides of these galleries, that receive light from above, are lined with elegant shops so that the gallery becomes like a city or even a small world in which the avid shopper will find everything they need" (in Benjamin, 2004, p. 69). Johann Friedrich Geist has systematized the great diversity in these kinds of galleries. From those of a single straight, perpendicular corridor to those organized in a cross in relation to the number of corridors. Or from the gallery corridors to the corridor-plaza galleries or gallery-plaza, passing through the bodies of buildings at an angle, two angles or more, or like galleries of one, two and four entrance/facades, among other kinds (Geist, 1989). Depending on the country, the language and certain traditions, the French term passage has been translated as pasaje, arcade, galerie, galería, passaz and galleria, among others. In the case of Peru, however, the week institutionalization of the passage has produced a ambiguous meaning for the term that is identified more as a building of commercial use, with a kind of narrow pedestrian street and almost exclusively residential use. The text will use the term gallery in its classical commercial sense.

2 Referring to a Andean-urban mixture of any style, function or use.

3 The differences that Kurt Weyland (1997) establishes are accurate between the classical notions of liberalism and populism and those defined as neo-liberalism and neo-populism. Unlike classical liberalism that was applied to create a State, neo-liberalism seeks seeks to undo the state apparatus through the absolutism of the free market laws and the privatization of the entire production system. Unlike the classical populism of the mid-twentieth century that promoted indusrial modernization based itself on the emerging Latin American industrial proletariat, the neo-populism of the 1990's retains a different program and social reference: a commitment to the policies of the free market and economica transnationalization. It opposes organized forms of civil society and evokes a populist rhetoricto the informal sector and the mass of the poor in extreme situations as its principal social base.

In Weyland's terms, Fujimori is a typical neo-populist neo-liberal (Weyland, 1997).

4 Regarding the origens of the passage, Geist identifies five kinds of bazar, from the regular bazar-corridor to the kind of labyrinthine bazar-city(Geist, 1989). However, the gallery is constituted as a space of redefinition of a preexiting tradition, embodying its own singular typological notation in its connection with the transformations brought on by industrial capitlaism beginning in the eigh teenth century and the corresponding project of modern cities and societies: this is its singularity as a componend and essential magic box of this new world. Expressed in Rolf Tiedemann's terms, for Walter Benjamin, the galleries must be conceived not only as mediums of production that must acheive a certain function within the industrial capitalist modernity, but as architectonice devices that embody the iconic fantasy of an unconscious collective that is anxious to surpass the limits of history (Benjamin, 1982, p. 14). This is the western gallery converted into a muth of modernity and omnipresent temple of a new religion where merchandise is deified, arcvhitecture is a magic space and eerie landscape to discover ephimeral pleasure.

5 Peruvian expression to describe a person that "pretends to be elegant and refined" (Editor's note). 6 After the earthquake of 1970 , the glass roof was re- moved for security reasons, giving the gallery architecture a look of being abandoned. The naked roof has been a symbol of the incongruencies of an architecture conceived for the cold and rain in a place where it is neither cold nor rains.

7 The extensive demolition proposals of the colonial heritage, as well as the widening of downtown streets and the massive construction of blocks formed by the Pilot Plan of 1949 failed to materialize its radicality. However, the center would not be the same space after the widening the roads and the construction of modern towers at the beginning of the fifties.

8 The commercial Gamarra neighborhood is located in the traditional central district of La Victoria. It occupies a variable extension of more than 63 blocks in middle of the urban inferno that still represents the mythical commercial neighborhood of La Parada. Today, with a movement of more than 600 million dollars a year, this emporium of hundreds of small businesses symbolizes the popular enterprise and the strength of popular capitalism. Gamarra is a fragment of the informal commercial neighborhood that has developed its own capacities of global insertion in middle of an ever more adverse market. As Miriam Chion argues, it is an exemplary case of the capacity of a marginal area to develop the appropriate institutional resources that have allowed it to connect with metropolitan and international networks (2002). 9 The market grounds were formed as a jumble of narrow corridors no more than $2 \mathrm{~m}$ wide and hundreds of tiny stalls $2 \times 3 \mathrm{~m}$. Covered with plastic awnings and other precarious materials, these markets were converted into a manifest symbol of a society that, like the Peruvian in the eighties, was plunged into the worst economic and political crisis in its history. Those located in the historical center were dismantled and relocated at the edge of the central area halfway through the nineties, giving rise to new popular commercial centers.

10 It should be recognized that in this first stage the climatic reason could have been influenced in the weak implementation of the Lima galleries of the nineties. In cities without rain or extreme weather, is at least one of the main reasons for the success of the passage: to be an welcome temporary refuge from the sudden downpour of inclement weather in the city (Benjamin, 1982). The first galleries in Lima had no alternative other than to recognize that the exterior city continues to be the privileged space of consumption and the exhilirating showcase of a tropical flâneur anxious to see and be seen. ARQ

WILEY LUDEÑA | Architect, Universidad Ricardo Palma, 1978; Master of Arquitectura, Universidad Nacional de Ingeniería, 1990 and Doctor in Urbanism, Technische Universität Hamburg-Harburg, 1996. His work as theorist and historian consists of the study of formation patterns in the neighborhoods of the Peruvian and Latin American city of the 19th century and the first half of the 20th century, and the analysis of uses and transformation of these public spaces in the cities of Peru during the Republican period. Since 1980 he has been the journalist critic of architecture in the Peruvian press. He is the founder and director of the Master in History and Criticism of Architecture and the Master of Urban Renovation of the Universidad Nacional de Ingeniería. He currently is a professor at the Universidad Nacional de Ingeniería and the Pontificia Universidad Católica del Perú.

DIANA TORRES | Architect, Universidad Nacional de Ingeniería, 2013. Coordinator of the Editorial Fund of Architecture, Urbanism and Arts of the Universidad Nacional de Ingeniería. She works in the technical direction of human settlements and land use of the Municipalidad Metropolitana de Lima. Her area of study centers around the urban dynamic in the peripheries of the city of Lima. She also currently teaches at the Pontificia Universidad Católica del Perú. 